\title{
PLATES WITH INCOMPATIBLE PRESTRAIN
}

\author{
KAUSHIK BHATTACHARYA, MARTA LEWICKA AND MATHIAS SCHÄFFNER
}

\begin{abstract}
We study the effective elastic behavior of incompatibly prestrained plates, where the prestrain is independent of thickness as well as uniform through the thickness. We model such plates as three-dimensional elastic bodies with a prescribed pointwise stress-free state characterized by a Riemannian metric $G$ with the above properties, and seek the limiting behavior as the thickness goes to zero.

We first establish that the $\Gamma$-limit is a Kirchhoff-type bending theory when the energy per volume scales as the second power of thickness. We then show the somewhat surprising result that there are metrics which are not immersible, but have zero bending energy. This implies that there are a hierarchy of plate theories for such prestrained plates. We characterize the non-immersible metrics that have zero bending energy (if and only if the Riemann curvatures $R_{112}^{3}, R_{221}^{3}$ and $R_{1212}$ of $G$ do not identically vanish), and illustrate them with examples. Of particular interest is an example where $G_{2 \times 2}$, the two-dimensional restriction of $G$ is flat, but the plate still has non-trivial energy that scales similar to Föppl - von Kármán plates. Finally, we apply these results to a model of nematic glass, including a characterization of the condition when the metric is immersible, for $G=\mathrm{Id}_{3}+\gamma \vec{n} \otimes \vec{n}$ given in terms of the inhomogeneous unit director field distribution $\vec{n} \in \mathbb{R}^{3}$.
\end{abstract}

\section{INTRODUCTION}

There are a number of phenomena where thin plates become prestrained in an inhomogeneous and incompatible manner. In such situations, the plates become internally stressed, deform out of plane and assume non-trivial three dimensional shapes. Growing leaves, gels subjected to differential swelling, electrodes in electrochemical cells, edges of torn plastic sheets are but a few examples (see [11, 14] and referenced there). It has also been recently suggested that such incompatible prestrains may be exploited as means of actuation of micro-mechanical devices [18, 19.

The Föppl - von Kármán plate theory has been widely used in the literature to study incompatible pre-strained-induced bending. It has recently been shown [14 that such a theory arises as a Gamma-limit of the three-dimensional energy if the prestrain identity tends to zero together with the plate's thickness. However, there are situations like those of liquid crystalline solids where the pre-strain is not small. Therefore, a proper formulation of such problems is of interest.

A possible mathematical foundation relies on a model referred to as "non-Euclidean" theory of elasticity. This model postulates that the three dimensional elastic body seeks to realize a configuration with a prescribed Riemannian metric $G$. Although there always exists a Lipschitz isometric immersion of any $G$ [7], any such immersion is necessarily neither orientation preserving nor reversing in any neighborhood of a point where the Riemann curvature of $G$ does not vanish (i.e. when the metric is non-Euclidean). Excluding such unphysical deformations leads to the model potential (or elastic) energy $E$ which measures how far a given deformation $u$ is from being an orientation preserving realization of $G$. The infimum of $E$ in absence of any forces or boundary conditions is indeed strictly positive for any non-Euclidean $G$ [13], and this points to

Date: November 19, 2014. 
the existence of non-zero stress at free equilibria. Of particular interest are situations where the domain occupied by the body is small in one dimension compared to the other two. In such situations, it is of interest to establish asymptotic models.

To be specific, let $\Omega \subset \mathbb{R}^{2}$ be an open, bounded, smooth and simply connected set. For small $h>0$ we consider thin plates with mid-plate $\Omega$, given by:

$$
\Omega^{h}=\Omega \times\left(-\frac{h}{2}, \frac{h}{2}\right)=\left\{x=\left(x^{\prime}, x_{3}\right) ; x^{\prime} \in \Omega,\left|x_{3}\right|<\frac{h}{2}\right\} .
$$

Let $G: \bar{\Omega} \rightarrow \mathbb{R}^{3 \times 3}$ be a smooth field of symmetric positive definite matrices, so that it defines a Riemannian metric on $\Omega^{h}$. In this paper we study the situation where $G$ is independent of the thickness as well and uniform through the thickness. Specifically, we assume

$$
G\left(x^{\prime}, x_{3}\right)=G\left(x^{\prime}\right)=\left[G_{i j}\left(x^{\prime}\right)\right]_{i, j=1 \ldots 3} \quad \forall\left(x^{\prime}, x_{3}\right) \in \Omega^{h} .
$$

We consider the following "non-Euclidean energy" functional:

$$
E^{h}\left(u^{h}\right)=\frac{1}{h} \int_{\Omega^{h}} W\left(\nabla u^{h} A^{-1}\right) \mathrm{d} x \quad \forall u^{h} \in W^{1,2}\left(\Omega^{h}, \mathbb{R}^{3}\right),
$$

where $A$ is the positive definite symmetric square root of $G$ :

$$
A=\sqrt{G},
$$

while $W: \mathbb{R}^{3 \times 3} \longrightarrow \overline{\mathbb{R}}_{+}$is the elastic energy density. In addition to being $\mathcal{C}^{2}$ regular in a neighborhood of $S O(3)$, the density $W$ is assumed to satisfy the normalization, frame indifference and nondegeneracy conditions as below:

$$
\begin{aligned}
& \exists c>0 \quad \forall F \in \mathbb{R}^{3 \times 3} \quad \forall R \in S O(3) \quad W(R)=0, \quad W(R F)=W(F), \\
& W(F) \geq c \operatorname{dist}^{2}(F, S O(3)) .
\end{aligned}
$$

We are interested in understanding the limiting behavior of inf $E^{h}$ as $h \rightarrow 0$.

It follows from the classical work of Nash and Kuiper [21, 22], and from Gromov's convex integration method [7, that any smooth metric $G_{2 \times 2}$ on $\Omega$ has a $\mathcal{C}^{1, \alpha}$ (with $\alpha<1 / 7$ ) isometric immersion in $\mathbb{R}^{3}$ (see also [2, 4]). In view of the work of Le Dret and Raoult [12, if this immersion is smooth, we expect that the highest non-zero scaling of the energy $E^{h}$ is $\frac{1}{h^{2}} E^{h}$ (in fact this is shown in Corollary 3.2 below, with the critical regularity $W^{2,2}$ ). Therefore, we focus on this and smaller scaling of the energy.

We explore three issues.

First, in Part A, we establish (Theorems 2.1] and 3.1) that the $\Gamma$-limit of $\frac{1}{h^{2}} E^{h}$ is given by a Kirchhoff bending energy functional (2.5) acting on the set of all $W^{2,2}$ realizations of $G_{2 \times 2}$ in $\mathbb{R}^{3}$. The main tool is the geometric rigidity theorem of Friesecke, James and Müller [6]. In fact, these authors used this theorem to rigorously derive the nonlinear Kirchhoff bending theory as the $\Gamma$-limit of the classical Euclidean elasticity theory $\left(G=\operatorname{Id}_{3}\right)$ under the assumption that $E^{h}$ scales like $h^{2}$. Lewicka and Pakzad [13] extended their approach to the non-Euclidean setting in the particular case $G=G_{2 \times 2}^{*}+e_{3} \otimes e_{3}$. Our results extend this to the case of arbitrary $G$ that satisfy (1.1).

Second, in Part B, we show that the situation (1.1) can lead to other non-trivial theories. We first show (Theorems 5.3 and 5.5) that the limit of the infimum of $\frac{1}{h^{2}} E^{h}$ is non-zero if and only if the three Riemann curvatures $R_{112}^{3}, R_{221}^{3}$ and $R_{1212}$ of $G$ do not vanish identically. Therefore, there exist non-immersable metrics $G$ for whom the minimum of their bending energy is zero. Therefore, 
these metrics lead to non-trivial theories at scalings smaller than $h^{2}$. While the detailed derivation of such theories remain open, we explore them through Examples 6.1 through 6.5. We show in Example6.1 that the infimum of $E^{h}$ scales as $h^{4}$ (Theorem 7.1). This is reminiscent of the scaling of Föppl - von Kármán theories. However, in this example $G_{2 \times 2}$ is flat and therefore the limiting deformation involves no bending! In other words, these can be regarded as a generalized plane stress with Föppl - von Kármán scaling. Further, we show in Example6.5 that this residual energy is not limited to cases where $G_{2 \times 2}$ is flat. In other words, there are situations when one has large incompatible pre-strain, Föppl - von Kármán or milder bending. A complete characterization of these theories remain an ongoing endeavor.

Third, in Part C, we apply our results to liquid crystal glass where metric $G$ of the form $\operatorname{Id}_{3}+\gamma \vec{n} \otimes \vec{n}$ for unitary director field $\vec{n} \in \mathbb{R}^{3}$ and a constant parameter $\gamma$. It has recently been suggested that such metrics and the resulting deformation be exploited as means of actuation of micro-mechanical devices [18, 19]. We show in Theorem 8.1 that this metric is immersible if and only if $\operatorname{curl}^{T} \operatorname{curl}(\vec{n} \otimes \vec{n})=0$. Further, for the general three dimensional case we show in Theorem 8.5 that the $\Gamma$-limit energy measures the bending content of the form: $\left(\operatorname{Id}_{2}-\tilde{\gamma} n \otimes n\right) F_{2 \times 2}\left(\operatorname{Id}_{2}-\right.$ $\tilde{\gamma} n \otimes n)$, where $n$ is the in-plane component of $\vec{n}$ and $\tilde{\gamma}$ is an explicitly given inhomogeneous parameter.

The paper is organized as follows. Part A consists of three sections. We prove the lower bound to $\frac{1}{h^{2}} E^{h}$ in section 2 and the upper bound in section 3 . section 4 specializes the formulas of the bending energy to the isotropic case. Part B also consists of three sections. In section 5, we derive equivalent conditions for the scaling $E^{h} \sim h^{2}$ to be optimal. We provide examples of the non-trivial (flat or non-flat) limiting configurations in section 6 . In section 7 we show the equivalent condition for optimality of the scaling $E^{h} \sim h^{4}$, in a particular case pertaining to one of the examples. The application to nematic elastomers is in section 8 which is the sole section in Part C.

Throughout the paper, we use the following notation. Given a matrix $F \in \mathbb{R}^{3 \times 3}$ we denote its trace by: $\operatorname{tr} F$, its transpose by: $F^{T}$, its symmetric part by: $\operatorname{sym} F=\frac{1}{2}\left(F+F^{T}\right)$, and its skew part by: skew $F=F-\operatorname{sym} F$. We shall use the matrix norm $|F|=\left(\operatorname{tr}\left(F^{T} F\right)\right)^{1 / 2}$, which is induced by the inner product $\left\langle F_{1}: F_{2}\right\rangle=\operatorname{tr}\left(F_{1}^{T} F_{2}\right)$. The $k \times l$ principal minor of a matrix $F \in \mathbb{R}^{3 \times 3}$ will be denoted by $F_{k \times l}$. Conversely, for a given $F_{k \times l} \in \mathbb{R}^{k \times l}$, the $3 \times 3$ matrix with

principal minor equal $F_{k \times l}$ and all other entries equal to 0 , will be denoted $F_{k \times l}^{*}$. All limits are taken as the thickness parameter $h$ vanishes, i.e. when $h \rightarrow 0$. Finally, by $C$ we denote any universal constant, independent of $h$.

Acknowledgments. M.L. was partially supported by the NSF Career grant DMS-0846996 and by the NSF grant DMS-1406730. K.B. was partially supported by the NSF PIRE grant OISE0967140.

\section{Part A: Bending limit}

\section{The Bending EnERGY: THE LOWER BOUnd}

We begin by characterizing sequences of deformations for which $E^{h} \leq h^{2}$ :

Theorem 2.1. For a given sequence of deformations $u^{h} \in W^{1,2}\left(\Omega^{h}, \mathbb{R}^{3}\right)$ satisfying:

$$
E^{h}\left(u^{h}\right) \leq C h^{2},
$$


where $C$ is a uniform constant, there exists a sequence of translations $c^{h} \in \mathbb{R}^{3}$, such that the following properties hold for the normalised deformations $y^{h} \in W^{1,2}\left(\Omega^{1}, \mathbb{R}^{3}\right)$ :

$$
y^{h}\left(x^{\prime}, x_{3}\right)=u^{h}\left(x^{\prime}, h x_{3}\right)-c^{h} .
$$

(i) There exists $y \in W^{2,2}\left(\Omega, \mathbb{R}^{3}\right)$ such that, up to a subsequence:

$$
y^{h} \rightarrow y \quad \text { strongly in } W^{1,2}\left(\Omega^{1}, \mathbb{R}^{3}\right) .
$$

(ii) The deformation y realizes the midplate metric:

$$
(\nabla y)^{T} \nabla y=G_{2 \times 2} .
$$

Consequently, the unit normal $\vec{N}$ to the surface $y(\Omega)$ and the Cosserat vector $\vec{b}$ below have the regularity $\vec{N}, \vec{b} \in W^{1,2} \cap L^{\infty}\left(\Omega, \mathbb{R}^{3}\right)$ :

$$
\vec{N}=\frac{\partial_{1} y \times \partial_{2} y}{\left|\partial_{1} y \times \partial_{2} y\right|} \quad \vec{b}=(\nabla y)\left(G_{2 \times 2}\right)^{-1}\left[\begin{array}{l}
G_{13} \\
G_{23}
\end{array}\right]+\frac{\sqrt{\operatorname{det} G}}{\sqrt{\operatorname{det} G_{2 \times 2}}} \vec{N} .
$$

(iii) Define the quadratic forms:

$$
\begin{array}{r}
\mathcal{Q}_{3}(F)=D^{2} W(\mathrm{Id})(F, F), \\
\mathcal{Q}_{2}\left(x^{\prime}, F_{2 \times 2}\right)=\min \left\{\mathcal{Q}_{3}\left({\sqrt{G\left(x^{\prime}\right)}}^{-1} \tilde{F}{\sqrt{G\left(x^{\prime}\right)}}^{-1}\right) ; \tilde{F} \in \mathbb{R}^{3 \times 3} \text { with } \tilde{F}_{2 \times 2}=F_{2 \times 2}\right\} .
\end{array}
$$

The form $\mathcal{Q}_{3}$ is defined for all $F \in \mathbb{R}^{3 \times 3}$, while $\mathcal{Q}_{2}\left(x^{\prime}, \cdot\right)$ are defined on $F_{2 \times 2} \in \mathbb{R}^{2 \times 2}$. Both forms $\mathcal{Q}_{3}$ and all $\mathcal{Q}_{2}$ are nonnegative definite and depend only on the symmetric parts of their arguments. We then have the lower bound:

$$
\liminf _{h \rightarrow 0} \frac{1}{h^{2}} E^{h}\left(u^{h}\right) \geq \mathcal{I}_{G}(y),
$$

where:

$$
\mathcal{I}_{G}(y)=\frac{1}{24} \int_{\Omega} \mathcal{Q}_{2}\left(x^{\prime},(\nabla y)^{T} \nabla \vec{b}\right) \mathrm{d} x^{\prime} .
$$

Remark 2.2. Consider a particular case, when the metric $G$ in (1.2) has the structure $G=$ $G_{2 \times 2}^{*}+e_{3} \otimes e_{3}$ as in [11, 13]. Then, likewise: $A=A_{2 \times 2}^{*}+e_{3} \otimes e_{3}$, and $A^{-1} e_{3}=G^{-1} e_{3}=e_{3}$. From the formula (2.3) it follows that: $\vec{b}=\vec{N}$, and so the asymptotic expansion of approximate minimizers of (1.2) is: $u^{h}\left(x^{\prime}, x_{3}\right) \approx y\left(x^{\prime}\right)+x_{3} \vec{N}\left(x^{\prime}\right)$. Also, directly from (2.4) we obtain:

$$
\begin{aligned}
& \mathcal{Q}_{2}\left(x^{\prime}, F_{2 \times 2}\right)=\mathcal{Q}_{2}^{0}\left(A_{2 \times 2}^{-1}\left(x^{\prime}\right) F_{2 \times 2} A_{2 \times 2}^{-1}\left(x^{\prime}\right)\right) \\
& \text { where } \mathcal{Q}_{2}^{0}\left(F_{2 \times 2}\right)=\min \left\{\mathcal{Q}_{3}(\tilde{F}) ; \tilde{F} \in \mathbb{R}^{3 \times 3} \text { with } \tilde{F}_{2 \times 2}=F_{2 \times 2}\right\} .
\end{aligned}
$$

Therefore, the limiting functional has the form:

$$
\mathcal{I}_{G}(y)=\frac{1}{24} \int_{\Omega} \mathcal{Q}_{2}^{0}\left(A_{2 \times 2}^{-1}(\nabla y)^{T} \nabla \vec{N} A_{2 \times 2}^{-1}\right) \mathrm{d} x^{\prime}
$$

and it depends on $y$ only through the second fundamental form $\Pi_{y}=(\nabla y)^{T} \nabla \vec{N}$ of the deformed mid-plate $y(\Omega)$. For the isotropic density $W$ (see (4.4)), one gets:

$$
\mathcal{Q}_{2}^{0}\left(F_{2 \times 2}\right)=\mathcal{Q}_{2, i s o}^{0}\left(F_{2 \times 2}\right)=\mu\left|\operatorname{sym} F_{2 \times 2}\right|^{2}+\frac{\lambda \mu}{\lambda+\mu}\left|\operatorname{tr} F_{2 \times 2}\right|^{2} .
$$

We see that we recover the results of [13] exactly. 
Before proving Theorem 2.1, we first state the approximation lemma from [13], which is just rephrasing Theorem 10 in [6] in the present non-Euclidean elasticity context.

Lemma 2.3. Assume (2.1). There exists matrix fields $Q^{h} \in W^{1,2}\left(\Omega, \mathbb{R}^{3 \times 3}\right)$ such that:

(i) $\frac{1}{h} \int_{\Omega^{h}}\left|\nabla u^{h}\left(x^{\prime}, x_{3}\right)-Q^{h}\left(x^{\prime}\right)\right|^{2} \mathrm{~d} x \leq C\left(h^{2}+\frac{1}{h} \int_{\Omega^{h}} \operatorname{dist}^{2}\left(\nabla u^{h} A^{-1}, S O(3)\right) \mathrm{d} x\right)$,

(ii) $\int_{\Omega}\left|\nabla Q^{h}\left(x^{\prime}\right)\right|^{2} \mathrm{~d} x^{\prime} \leq C\left(1+\frac{1}{h^{3}} \int_{\Omega^{h}} \operatorname{dist}^{2}\left(\nabla u^{h} A^{-1}, S O(3)\right) \mathrm{d} x\right)$.

\section{Proof of Theorem 2.1.}

1. By Lemma 2.3 we see that the sequence $\left\{Q^{h}\right\}$ is bounded in $L^{2}$, together with its derivatives. Therefore, up to a subsequence:

$$
Q^{h} \rightarrow Q \quad \text { weakly in } W^{1,2}\left(\Omega, \mathbb{R}^{3 \times 3}\right) .
$$

Consider the rescaled deformations $y^{h} \in W^{1,2}\left(\Omega^{1}, \mathbb{R}^{3}\right)$ given by:

$$
y^{h}\left(x^{\prime}, x_{3}\right)=u^{h}\left(x^{\prime}, h x_{3}\right)-f_{\Omega^{h}} u^{h} .
$$

Since:

$$
\int_{\Omega^{1}}\left|\nabla u^{h}\left(x^{\prime}, h x_{3}\right)-Q\left(x^{\prime}\right)\right|^{2} \leq 2 \int_{\Omega^{1}}\left|\nabla u^{h}\left(x^{\prime}, h x_{3}\right)-Q^{h}\right|^{2}+2 \int_{\Omega}\left|Q^{h}-Q\right|^{2},
$$

it follows by Lemma 2.3 (i) and (2.7) that:

$$
\left[\begin{array}{lll}
\partial_{1} y^{h} & \partial_{2} y^{h} & \frac{\partial_{3} y^{h}}{h}
\end{array}\right] \rightarrow Q \quad \text { strongly in } L^{2}\left(\Omega^{1}, \mathbb{R}^{3 \times 3}\right) .
$$

In particular, the sequence $\left\{\nabla y^{h}\right\}$ is bounded in $L^{2}$. Since $f y^{h}=0$, by the Poincaré inequality, a subsequence of $\left\{y^{h}\right\}$ converges weakly in $W^{1,2}\left(\Omega^{1}, \mathbb{R}^{3}\right)$ to some limiting field $y$. On the other hand, $\left\{\nabla y^{h}\right\}$ converge strongly because of (2.8):

$$
\nabla_{\tan } y^{h} \rightarrow Q_{3 \times 2} \text { and } \partial_{3} y^{h} \rightarrow 0 \quad \text { strongly in } L^{2}\left(\Omega^{1}\right) .
$$

Consequently, the convergence of $\left\{y^{h}\right\}$ is actually strong, and $y=y\left(x^{\prime}\right) \in W^{2,2}\left(\Omega, \mathbb{R}^{3}\right)$ with:

$$
\nabla y=\nabla_{\tan } y=Q_{3 \times 2} \text {. }
$$

We have thus proved (i) in Theorem 2.1

2. Note that by Lemma 2.3 (i):

$$
\begin{aligned}
& \int_{\Omega} \operatorname{dist}^{2}\left(Q^{h} A^{-1}, S O(3)\right) \mathrm{d} x^{\prime} \\
& \quad \leq \frac{C}{h}\left(\int_{\Omega^{h}} \operatorname{dist}^{2}\left(\nabla u^{h} A^{-1}, S O(3)\right)+\int_{\Omega^{h}}\left|\nabla u^{h}\left(x^{\prime}, x_{3}\right)-Q^{h}\left(x^{\prime}\right)\right|^{2} \mathrm{~d} x\right) \leq C h^{2} .
\end{aligned}
$$

Therefore, by (2.7):

$$
Q A^{-1} \in S O(3) \quad \forall \text { a.e. } x^{\prime} \in \Omega,
$$

so, in particular, we obtain (2.2), and automatically:

$$
\nabla y \in W^{1,2} \cap L^{\infty}\left(\Omega, \mathbb{R}^{3}\right) .
$$


Further, by (2.2) and using the formula $a \times(b \times c)=\langle a, c\rangle b-\langle a, b\rangle c$, one gets:

$$
\begin{aligned}
\left|\partial_{1} y \times \partial_{2} y\right|^{2} & =\left|A e_{1} \times A e_{2}\right|^{2}=\left\langle A e_{1},\left(A e_{1} \times A e_{2}\right) \times A e_{1}\right\rangle \\
& =\left\langle A e_{2},\left\langle A e_{1}, A e_{1}\right\rangle A e_{2}-\left\langle A e_{1}, A e_{1}\right\rangle A e_{1}\right\rangle \\
& =G_{11} G_{22}-G_{12}^{2}=\operatorname{det} G_{2 \times 2} .
\end{aligned}
$$

Hence, in view of (2.12) $: \vec{N} \in W^{1,2} \cap L^{\infty}$ and, consequently, the same holds for $\vec{b}$.

3. We will now prove that, assuming (2.9) and (2.2), condition (2.11) is equivalent to $\vec{b}=Q e_{3}$ satisfty (2.3). Indeed, write:

$$
\vec{b}=\alpha_{1} \partial_{1} y+\alpha_{2} \partial_{2} y+\alpha_{3} \vec{N} .
$$

By (2.13), we obtain:

$$
\operatorname{det} Q=\operatorname{det}\left[\begin{array}{lll}
\partial_{1} y & \partial_{2} y & \alpha_{3} \vec{N}
\end{array}\right]=\alpha_{3}\left|\partial_{1} y \times \partial_{2} y\right|=\alpha_{3} \sqrt{\operatorname{det} G_{2 \times 2}} .
$$

Now, (2.11) is equivalent to $Q^{T} Q=G$ and $\operatorname{det} Q>0$, hence (2.11) is further equivalent to:

$$
\begin{aligned}
G_{13} & =\left\langle\vec{b}, \partial_{1} y\right\rangle=\alpha_{1} G_{11}+\alpha_{2} G_{12} \\
G_{23} & =\left\langle\vec{b}, \partial_{2} y\right\rangle=\alpha_{1} G_{21}+\alpha_{2} G_{22} \\
\sqrt{\operatorname{det} G} & =\operatorname{det} Q=\alpha_{3} \sqrt{\operatorname{det} G_{2 \times 2}},
\end{aligned}
$$

which yields:

$$
\left[\begin{array}{l}
\alpha_{1} \\
\alpha_{2}
\end{array}\right]=\left(G_{2 \times 2}\right)^{-1}\left[\begin{array}{l}
G_{13} \\
G_{23}
\end{array}\right], \quad \alpha_{3}=\frac{\sqrt{\operatorname{det} G}}{\sqrt{\operatorname{det} G_{2 \times 2}}},
$$

exactly as claimed in (2.3).

4. We now modify the sequence $\left\{Q^{h}\right\}$ to another sequence $\tilde{Q}^{h} \in L^{2}\left(\Omega, \mathbb{R}^{3 \times 3}\right)$ so that:

$$
R^{h}=\tilde{Q}^{h} A^{-1} \in S O(3) \quad \forall \text { a.e. } x^{\prime} \in \Omega,
$$

This is done by projecting $\mathbb{P}_{S O(3)}$ onto $S O(3)$ when possible, and setting:

$$
\tilde{Q}^{h} A^{-1}= \begin{cases}\mathbb{P}_{S O(3)}\left(Q^{h} A^{-1}\right) & \text { if } Q^{h} A^{-1} \in \mathcal{O}_{\epsilon}(S O(3)) \\ \operatorname{Id} & \text { otherwise }\end{cases}
$$

with a small $\epsilon>0$. Then, by (2.10):

$$
\int_{\Omega}\left|\tilde{Q}^{h}-Q^{h}\right|^{2} \leq C \int_{\Omega}\left|\tilde{Q}^{h} A^{-1}-Q^{h} A^{-1}\right|^{2} \leq C \int_{\Omega} \operatorname{dist}^{2}\left(Q^{h} A^{-1}, S O(3)\right) \leq C h^{2} .
$$

In particular, by (2.7):

$$
\tilde{Q}^{h} \rightarrow Q \quad \text { strongly in } L^{2}\left(\Omega, \mathbb{R}^{3 \times 3}\right) .
$$

Define the scaled strains $S^{h} \in L^{2}\left(\Omega^{1}, \mathbb{R}^{3 \times 3}\right)$ by:

$$
S^{h}\left(x^{\prime}, x_{3}\right)=\frac{1}{h}\left(\left(R^{h}\right)^{T} \nabla u^{h}\left(x^{\prime}, h x_{3}\right) A^{-1}-\mathrm{Id}\right) .
$$

We have, in view of Lemma 2.3 (i) and (2.14):

$$
\begin{aligned}
\int_{\Omega^{1}}\left|S^{h}\right|^{2} & \leq \frac{C}{h^{2}} \int_{\Omega^{1}}\left|\nabla u^{h}\left(x^{\prime}, h x_{3}\right)-\tilde{Q}^{h}\right|^{2} \mathrm{~d} x \\
& \leq \frac{C}{h^{3}} \int_{\Omega^{h}}\left|\nabla u^{h}-Q^{h}\right|^{2}+\frac{C}{h^{2}} \int_{\Omega}\left|Q^{h}-\tilde{Q}^{h}\right|^{2} \leq C,
\end{aligned}
$$


and hence a subsequence of $\left\{S^{h}\right\}$ converges:

$$
S^{h} \rightarrow \bar{S} \quad \text { weakly in } L^{2}\left(\Omega^{1}, \mathbb{R}^{3 \times 3}\right) .
$$

5. We now derive the formula on the limiting strain $\bar{S}$. Consider the difference quotients:

$$
f^{s, h}\left(x^{\prime}, x_{3}\right)=\frac{1}{h} \frac{1}{s}\left(y^{h}\left(x^{\prime}, x_{3}+s\right)-y^{h}\left(x^{\prime}, x_{3}\right)\right) \in L^{2}\left(\Omega^{1}, \mathbb{R}^{3 \times 3}\right) .
$$

By (2.8), it follows that:

$$
f^{s, h}\left(x^{\prime}, x_{3}\right)=\frac{1}{h} f_{0}^{s} \partial_{3} y^{h}\left(x^{\prime}, x_{3}+t\right) \mathrm{d} t \rightarrow \vec{b}\left(x^{\prime}\right) \quad \text { in } L^{2}\left(\Omega^{1}, \mathbb{R}^{3}\right) .
$$

Similarly:

$$
\partial_{3} f^{s, h}\left(x^{\prime}, x_{3}\right)=\frac{1}{s}\left(h^{-1} \partial_{3} y^{h}\left(x^{\prime}, x_{3}+s\right)-h^{-1} \partial_{3} y^{h}\left(x^{\prime}, x_{3}\right)\right) \rightarrow 0 \quad \text { strongly in } L^{2}\left(\Omega^{1}, \mathbb{R}^{3}\right),
$$

while for $i=1,2$, by (2.15) and (2.17):

$$
\begin{aligned}
\partial_{i} f^{s, h}\left(x^{\prime}, x_{3}\right)= & \frac{1}{h} \frac{1}{s}\left(\nabla u^{h}\left(x^{\prime}, h\left(x_{3}+s\right)\right)-\nabla u^{h}\left(x^{\prime}, h x_{3}\right)\right) e_{i} \\
= & \frac{1}{s} R^{h}\left(x^{\prime}\right)\left(S^{h}\left(x^{\prime}, x_{3}+s\right)-S^{h}\left(x^{\prime}, x_{3}\right)\right) A e_{i} \\
& \quad \rightarrow \frac{1}{s} Q A^{-1}\left(\bar{S}\left(x^{\prime}, x_{3}+s\right)-\bar{S}\left(x^{\prime}, x_{3}\right)\right) A e_{i} \quad \text { weakly in } L^{2}\left(\Omega^{1}, \mathbb{R}^{3}\right) .
\end{aligned}
$$

Concluding:

and hence:

$$
f^{s, h} \rightarrow \vec{b} \quad \text { weakly in } W^{1,2}\left(\Omega^{1}, \mathbb{R}^{3}\right),
$$

$$
\forall i=1,2 \quad \partial_{i} \vec{b}\left(x^{\prime}\right)=\frac{1}{s} Q A^{-1}\left(\bar{S}\left(x^{\prime}, x_{3}+s\right)-\bar{S}\left(x^{\prime}, x_{3}\right)\right) A e_{i} .
$$

By (2.11), $Q A^{-1}$ may be replaced by $Q^{T,-1} A$, so that:

$$
\forall i=1,2 \quad \bar{S}\left(x^{\prime}, x_{3}+s\right) A e_{i}=\bar{S}\left(x^{\prime}, x_{3}\right) A e_{i}+s A^{-1} Q^{T} \partial_{i} \vec{b},
$$

and in view of (2.9) we obtain:

$$
\left(A \bar{S}\left(x^{\prime}, x_{3}+s\right) A\right)_{2 \times 2}=\left(A \bar{S}\left(x^{\prime}, x_{3}\right) A\right)_{2 \times 2}+s(\nabla y)^{T} \nabla \vec{b} .
$$

6. We now compute the lower bound on the rescaled energies. Define the 'good' sets:

$$
\Omega_{h}^{1}=\left\{\left(x^{\prime}, x_{3}\right) \in \Omega^{1} ;\left|S^{h}\left(x^{\prime}, x_{3}\right)\right|^{2} \leq \frac{1}{h}\right\} .
$$

In view of (2.16), it follows the convergence of characteristic functions:

$$
\chi_{h}=\chi_{\Omega_{h}^{1}} \rightarrow 1 \quad \text { strongly in } L^{1}\left(\Omega^{1}\right) .
$$

and therefore, by (2.17):

$$
\chi_{h} S^{h} \rightarrow \bar{S} \quad \text { weakly in } L^{2}\left(\Omega^{1}, \mathbb{R}^{3 \times 3}\right) .
$$

For small $h$, we may Taylor expand $W$ on the 'good' sets, using the definition of $S^{h}$ :

$$
\begin{aligned}
\forall\left(x^{\prime}, x_{3}\right) \in \Omega_{h}^{1} \quad \frac{1}{h^{2}} W\left(\nabla u^{h}\left(x^{\prime}, h x_{3}\right) A^{-1}\right) & =\frac{1}{h^{2}} W\left(\operatorname{Id}+h S^{h}\left(x^{\prime}, x_{3}\right)\right) \\
& =\frac{1}{2} \mathcal{Q}_{3}\left(S^{h}\left(x^{\prime}, x_{3}\right)\right)+o\left(\left|S^{h}\right|^{2}\right) .
\end{aligned}
$$


By (2.19), we now obtain:

$$
\begin{aligned}
\liminf _{h \rightarrow 0} \frac{1}{h^{2}} E^{h}\left(u^{h}\right) & \geq \liminf _{h \rightarrow 0} \frac{1}{h^{2}} \int_{\Omega_{h}^{1}} W\left(\nabla u^{h}\left(x^{\prime}, h x_{3}\right) A^{-1}\right) \mathrm{d} x \\
& =\liminf _{h \rightarrow 0} \frac{1}{2} \int_{\Omega^{1}} \mathcal{Q}_{3}\left(\chi_{h} S^{h}\left(x^{\prime}, x_{3}\right)\right) \geq \frac{1}{2} \int_{\Omega^{1}} \mathcal{Q}_{3}(\bar{S}) .
\end{aligned}
$$

Since the quadratic form $\mathcal{Q}_{3}$ is nonnegative definite, we obtain:

$$
\begin{aligned}
\frac{1}{2} \int_{\Omega^{1}} \mathcal{Q}_{3}(\bar{S}) & \geq \frac{1}{2} \int_{\Omega^{1}} \mathcal{Q}_{2}\left(\left(A \bar{S}\left(x^{\prime}, x_{3}\right) A\right)_{2 \times 2}\right) \\
& \left.=\frac{1}{2} \int_{\Omega} \mathcal{Q}_{2}\left(\left(A \bar{S}\left(x^{\prime}, 0\right) A\right)_{2 \times 2}\right) \mathrm{d} x^{\prime}+\frac{1}{2}\left(\int_{-1 / 2}^{1 / 2} s^{2} \mathrm{~d} s\right) \int_{\Omega} \mathcal{Q}_{2}(\nabla y)^{T} \nabla \vec{b}\right) \mathrm{d} x^{\prime} \\
& \left.\geq \frac{1}{24} \int_{\Omega} \mathcal{Q}_{2}(\nabla y)^{T} \nabla \vec{b}\right) \mathrm{d} x^{\prime}=\mathcal{I}_{G}(y),
\end{aligned}
$$

where we used (2.18). In view of (2.20), the proof is complete.

\section{The Bending energy: Recovery Sequence And the upper bound}

In this section we prove that the lower bound in Theorem 2.1] is optimal, in the following sense:

Theorem 3.1. For every isometric immersion $y \in W^{2,2}\left(\Omega, \mathbb{R}^{3}\right)$ of the metric $G_{2 \times 2}$ as in (2.2), there exists a sequence of 'recovery deformations' $u^{h} \in W^{1,2}\left(\Omega^{h}, \mathbb{R}^{3}\right)$, such that:

(i) The rescaled sequence $y^{h}\left(x^{\prime}, x_{3}\right)=u^{h}\left(x^{\prime}, h x_{3}\right)$ converges in $W^{1,2}\left(\Omega^{1}, \mathbb{R}^{3}\right)$ to $y$.

(ii) One has:

$$
\lim _{h \rightarrow 0} \frac{1}{h^{2}} E^{h}\left(u^{h}\right)=\mathcal{I}_{G}(y),
$$

where the Cosserat vector $\vec{b}$ in the definition (2.5) of the functional $\mathcal{I}_{G}$ is derived by (2.3).

It immediately follows that:

Corollary 3.2. Existence of a $W^{2,2}$ regular isometric immersion of the Riemannian metric $G_{2 \times 2}$ on $\Omega$ in $\mathbb{R}^{3}$ is equivalent to the upper bound on the energy scaling at minimizers:

$$
\exists C>0 \quad \inf _{u \in W^{1,2}\left(\Omega^{h}, \mathbb{R}^{3}\right)} E^{h}(u) \leq C h^{2} .
$$

Corollary 3.3. The limiting functional $\mathcal{I}_{G}$ attains its minimum.

Proof. Let $\left\{y_{n}\right\}_{n=1}^{\infty}$ be a minimizing sequence of $\mathcal{I}_{G}$. By Theorem 3.1, there exists sequences $u_{n}^{h} \in W^{1,2}\left(\Omega^{h}, \mathbb{R}^{3}\right)$ such that: $\lim _{h \rightarrow 0} u_{n}^{h}\left(x^{\prime}, h x_{3}\right)=y_{n}$ in $W^{1,2}\left(\Omega^{1}, \mathbb{R}^{3}\right)$ and $\lim _{h \rightarrow 0} \frac{1}{h^{2}} E^{h}\left(u_{n}^{h}\right)=$ $\mathcal{I}_{G}\left(y_{n}\right)$, for every $n$. Taking $u^{h}=u_{n}^{h(n)}$ for a sequence $h(n)$ converging to 0 as $n \rightarrow \infty$ sufficiently fast, we obtain: $E^{h}\left(u^{h}\right) \leq C h^{2}$. Therefore, by Theorem 2.1 there exists a limiting deformation $y \in W^{2,2}\left(\Omega, \mathbb{R}^{3}\right)$ so that:

$$
\mathcal{I}_{G}(y) \leq \liminf _{h \rightarrow 0} \frac{1}{h^{2}} E^{h}\left(u^{h}\right)=\lim _{n \rightarrow \infty} \mathcal{I}_{G}\left(y_{n}\right)=\inf \mathcal{I}_{G},
$$

which achieves that $y$ is a minimizer of $\mathcal{I}_{G}$. 
Before proving Theorem 3.1, recall that:

$$
\begin{aligned}
\forall F_{2 \times 2} \in \mathbb{R}_{\text {sym }}^{2 \times 2} \quad \mathcal{Q}_{2}\left(x^{\prime}, F_{2 \times 2}\right) & =\min \left\{\mathcal{Q}_{3}\left(A^{-1} \tilde{F} A^{-1}\right) ; \tilde{F} \in \mathbb{R}^{3 \times 3}, \tilde{F}_{2 \times 2}=F_{2 \times 2}\right\} \\
& =\min \left\{\mathcal{Q}_{3}\left(A^{-1}\left(F_{2 \times 2}^{*}+\operatorname{sym}\left(c \otimes e_{3}\right)\right) A^{-1}\right) ; c \in \mathbb{R}^{3}\right\} .
\end{aligned}
$$

In what follows, by:

$$
c\left(x^{\prime}, F_{2 \times 2}\right)
$$

we will denote the unique minimizer of the problem in (3.1).

\section{Proof of Theorem 3.1.}

1. Let $y \in W^{2,2}\left(\Omega, \mathbb{R}^{3}\right)$ satisfy (2.2). Define the Cosserat vector field $\vec{b} \in W^{1,2} \cap L^{\infty}\left(\Omega, \mathbb{R}^{3}\right)$ according to (2.3) and let:

$$
Q=\left[\begin{array}{lll}
\partial_{1} y & \partial_{2} y & \vec{b}
\end{array}\right] \in W^{1,2} \cap L^{\infty}\left(\Omega, \mathbb{R}^{3 \times 3}\right) .
$$

By Step 2 in the proof of Theorem 2.1, it follows that:

$$
Q A^{-1} \in S O(3) \quad \forall \text { a.e. } x^{\prime} \in \Omega \text {. }
$$

Define the limiting warping field $\vec{d} \in L^{2}\left(\Omega, \mathbb{R}^{3}\right)$ :

$$
\vec{d}\left(x^{\prime}\right)=Q^{T,-1}\left(c\left(x^{\prime},(\nabla y)^{T} \nabla \vec{b}\right)-\frac{1}{2} \nabla|\vec{b}|^{2}\right) .
$$

Let $\left\{d^{h}\right\}$ be a approximating sequence in $W^{1, \infty}\left(\Omega, \mathbb{R}^{3}\right)$, satisfying:

$$
d^{h} \rightarrow \vec{d} \quad \text { strongly in } L^{2}\left(\Omega, \mathbb{R}^{3}\right), \quad \text { and } \quad h\left\|d^{h}\right\|_{W^{1, \infty}} \rightarrow 0 .
$$

Note that such sequence can always be derived by reparametrizing (slowing down) a sequence of smooth approximations of $\vec{d}$. Similiarly, consider the approximations $y^{h} \in W^{2, \infty}\left(\Omega, \mathbb{R}^{3}\right)$ and $\vec{b}^{h} \in W^{1, \infty}\left(\Omega, \mathbb{R}^{3}\right)$, with the following properties:

$$
\begin{aligned}
& y^{h} \rightarrow y \quad \text { strongly in } W^{2,2}\left(\Omega, \mathbb{R}^{3}\right), \quad \text { and } \vec{b}^{h} \rightarrow \vec{b} \quad \text { strongly in } W^{1,2}\left(\Omega, \mathbb{R}^{3}\right) \\
& h\left(\left\|y^{h}\right\|_{W^{2, \infty}}+\left\|\vec{b}^{h}\right\|_{W^{1, \infty}}\right) \leq \epsilon \\
& \frac{1}{h^{2}}\left|\Omega \backslash \Omega_{h}\right| \rightarrow 0, \quad \text { where } \Omega_{h}=\left\{x^{\prime} \in \Omega ; y^{h}\left(x^{\prime}\right)=y\left(x^{\prime}\right) \text { and } \vec{b}^{h}\left(x^{\prime}\right)=\vec{b}\left(x^{\prime}\right)\right\}
\end{aligned}
$$

for some small $\epsilon>0$. Existence of approximations with the claimed properties follows by partition of unity and truncation arguments, as a special case of the Lusin-type result for Sobolev functions in [17] (see also Proposition 2 in [6]).

We now define $u^{h} \in W^{1, \infty}\left(\Omega^{h}, \mathbb{R}^{3}\right)$ by:

$$
u^{h}\left(x^{\prime}, x_{3}\right)=y^{h}\left(x^{\prime}\right)+x_{3} \vec{b}^{h}\left(x^{\prime}\right)+\frac{x_{3}^{2}}{2} d^{h}\left(x^{\prime}\right) .
$$

Consequently, the rescalings $y^{h} \in W^{1, \infty}\left(\Omega^{1}, \mathbb{R}^{3}\right)$ are:

$$
y^{h}\left(x^{\prime}, x_{3}\right)=y^{h}\left(x^{\prime}\right)+h x_{3} \vec{b}^{h}\left(x^{\prime}\right)+\frac{h^{2}}{2} x_{3}^{2} d^{h}\left(x^{\prime}\right),
$$

and therefore in view of (3.4) and (3.5), Theorem 3.1 (i) follows.:

2. Define the matrix fields:

$$
Q^{h}\left(x^{\prime}\right)=\left[\begin{array}{lll}
\partial_{1} y^{h} & \partial_{2} y^{h} & \vec{b}^{h}
\end{array}\right], \quad B^{h}\left(x^{\prime}\right)=\left[\begin{array}{lll}
\partial_{1} \vec{b}^{h} & \partial_{2} \vec{b}^{h} & d^{h}
\end{array}\right], \quad D^{h}\left(x^{\prime}\right)=\left[\begin{array}{lll}
\partial_{1} d^{h} & \partial_{2} d^{h} & 0
\end{array}\right],
$$


so that:

$$
\nabla u^{h}\left(x^{\prime}, x_{3}\right)=Q^{h}\left(x^{\prime}\right)+x_{3} B^{h}\left(x^{\prime}\right)+\frac{x_{3}^{2}}{2} D^{h}\left(x^{\prime}\right) \quad \forall\left(x^{\prime}, x_{3}\right) \in \Omega^{h} .
$$

Since $Q^{h}=Q$ in the set $\Omega_{h}$, then by (3.2) and the bound on the Lipschitz constants of $y^{h}$ and $\vec{b}^{h}$ in (3.5), we obtain:

$$
\operatorname{dist}\left(Q^{h} A^{-1}, S O(3)\right) \leq \frac{C}{h} \operatorname{dist}\left(x^{\prime}, \Omega_{h}\right) \leq \frac{C}{h}\left|\Omega \backslash \Omega_{h}\right|^{1 / 2} .
$$

The last bound above can be easily obtained by noting that if $B_{r}\left(x^{\prime}\right) \subset \Omega \backslash \Omega_{h}$ then $\pi r^{2} \leq\left|\Omega \backslash \Omega_{h}\right|$, which implies $r \leq C\left|\Omega \backslash \Omega_{h}\right|^{1 / 2}$. For $x^{\prime}$ close to the boundary of $\Omega$ one needs to slightly refine the argument using smoothness of $\partial \Omega$.

Consequently, by (3.6) and (3.5), it follows that for all $h$ sufficiently small:

$$
\begin{aligned}
\operatorname{dist}\left(\nabla u^{h}\left(x^{\prime}, h x_{3}\right)\right. & \left.A^{-1}, S O(3)\right) \leq \operatorname{dist}\left(Q^{h} A^{-1}, S O(3)\right)+h\left\|B^{h}\right\|_{L^{\infty}}+h^{2}\left\|D^{h}\right\|_{L^{\infty}} \\
\leq & \frac{C}{h}\left|\Omega \backslash \Omega_{h}\right|^{1 / 2}+C h\left(\left\|\nabla \vec{b}^{h}\right\|_{L^{\infty}}+\left\|d^{h}\right\|_{L^{\infty}}\right)+C h^{2}\left\|\nabla d^{h}\right\|_{L^{\infty}} \leq \epsilon_{0},
\end{aligned}
$$

where $\epsilon_{0}$ is such that the energy density $W$ is bounded and $\mathcal{C}^{2}$ regular in the neighbourhood $\mathcal{O}_{\epsilon_{0}}(S O(3))$. Taylor expanding $W$ at the given rotation in (3.2), we compute:

$$
\begin{aligned}
& \frac{1}{h^{2}} \int_{\Omega_{h} \times(-1 / 2,1 / 2)} W\left(\nabla u^{h}\left(x^{\prime}, h x_{3}\right) A^{-1}\right) \\
& \quad=\frac{1}{h^{2}} \int_{\Omega_{h} \times(-1 / 2,1 / 2)} W\left(\left(Q\left(x^{\prime}\right)+h x_{3} B^{h}\left(x^{\prime}\right)+h^{2} \frac{x_{3}^{2}}{2} D^{h}\left(x^{\prime}\right)\right) A^{-1}\right) \mathrm{d} x \\
& \quad=\frac{1}{2} \int_{\Omega_{h} \times(-1 / 2,1 / 2)} D^{2} W\left(Q\left(x^{\prime}\right) A^{-1}\right)\left(\left(x_{3} B^{h}\left(x^{\prime}\right)+h \frac{x_{3}^{2}}{2} D^{h}\left(x^{\prime}\right)\right) A^{-1}\right)^{\otimes 2}+\mathcal{O}(h) \mathrm{d} x .
\end{aligned}
$$

Also, by (3.5):

$$
\frac{1}{h^{2}} \int_{\left(\Omega \backslash \Omega_{h}\right) \times(-1 / 2,1 / 2)} W\left(\nabla u^{h}\left(x^{\prime}, h x_{3}\right) A^{-1}\right) \leq \frac{C}{h^{2}}\left|\Omega \backslash \Omega_{h}\right| \quad \rightarrow 0 .
$$

Hence:

$$
\begin{aligned}
\lim _{h \rightarrow 0} & \frac{1}{h^{2}} E^{h}\left(u^{h}\right)=\lim _{h \rightarrow 0} \frac{1}{h^{2}} \int_{\Omega_{h} \times(-1 / 2,1 / 2)} W\left(\nabla u^{h}\left(x^{\prime}, h x_{3}\right) A^{-1}\right) \\
& =\lim _{h \rightarrow 0} \frac{1}{2} \int_{\Omega_{h} \times(-1 / 2,1 / 2)} D^{2} W\left(Q A^{-1}\right)\left(\left(x_{3} B^{h}\left(x^{\prime}\right)+h \frac{x_{3}^{2}}{2} D^{h}\left(x^{\prime}\right)\right) A^{-1}\right)^{\otimes 2} \mathrm{~d} x \\
& =\lim _{h \rightarrow 0} \frac{1}{2} \int_{-1 / 2}^{1 / 2} \int_{\Omega_{h}} x_{3}^{2} D^{2} W\left(Q A^{-1}\right)\left(B^{h}\left(x^{\prime}\right) A^{-1}\right)^{\otimes 2} \mathrm{~d} x^{\prime} \mathrm{d} x_{3} \\
& =\lim _{h \rightarrow 0} \frac{1}{24} \int_{\Omega_{h}} \mathcal{Q}_{3}\left(\left(Q A^{-1}\right)^{T} B^{h}\left(x^{\prime}\right) A^{-1}\right) \\
& =\frac{1}{24} \int_{\Omega} \mathcal{Q}_{3}\left(A^{-1} Q^{T} B A^{-1}\right),
\end{aligned}
$$

where we have used the last convergence in (3.5), the frame invariance of the density function $W$ resulting in: $D^{2} W(R)(F, F)=D^{2} W(\mathrm{Id})\left(R^{T} F, R^{T} F\right)=\mathcal{Q}_{3}\left(R^{T} F\right)$ valid for all $R \in S O(3)$, and 
the following convergence:

$$
B^{h} \rightarrow B\left(x^{\prime}\right)=\left[\begin{array}{lll}
\partial_{1} \vec{b} & \partial_{2} \vec{b} & \vec{d}
\end{array}\right] \quad \text { strongly in } L^{2}\left(\Omega, \mathbb{R}^{3 \times 3}\right) .
$$

Now, note that by(3.3):

$$
\begin{aligned}
\operatorname{sym}\left(Q^{T} B\left(x^{\prime}\right)\right) & =\operatorname{sym}\left((\nabla y)^{T} \nabla \vec{b}\right)+\operatorname{sym}\left(e_{3} \otimes\left[\begin{array}{c}
(\nabla y)^{T} \vec{d}+\frac{1}{2} \nabla|\vec{b}|^{2} \\
\langle\vec{b}, \vec{d}\rangle
\end{array}\right]\right) \\
& =\operatorname{sym}\left((\nabla y)^{T} \nabla \vec{b}\right)+\operatorname{sym}\left(e_{3} \otimes c\left(x^{\prime},(\nabla y)^{T} \nabla \vec{b}\right)\right) .
\end{aligned}
$$

Therefore, (3.7) becomes:

$$
\lim _{h \rightarrow 0} \frac{1}{h^{2}} E^{h}\left(u^{h}\right)=\frac{1}{24} \int_{\Omega} \mathcal{Q}_{2}\left((\nabla y)^{T} \nabla \vec{b}\right) \mathrm{d} x^{\prime},
$$

achieving the proof of Theorem 3.1 .

\section{The effective Density $\mathcal{Q}_{2}$ And the Case of $W$ isotropic}

In this section, we further study the $2 \mathrm{~d}$ functional (2.5) and the inhomogeneous effective energy measure in (3.1). By $L_{3}: \mathbb{R}^{3 \times 3} \rightarrow \mathbb{R}^{3 \times 3}$ we denote the linear map with the property that:

$$
\mathcal{Q}_{3}(F)=\left\langle L_{3}(F): F\right\rangle \quad \text { and } \quad\left\langle L_{3}(F): \tilde{F}\right\rangle=\left\langle L_{3}(\tilde{F}): F\right\rangle \quad \forall F, \tilde{F} \in \mathbb{R}^{3 \times 3} .
$$

Note that by frame invariace of $W$ in (1.3) one has: $L_{3}(F)=L_{3}(\operatorname{sym} F)$ and $\operatorname{skew}\left(L_{3}(F)\right)=0$.

Lemma 4.1. Define the matrix field $M_{A}: \Omega \rightarrow \mathbb{R}^{3 \times 3}$ by:

$$
\forall i: 1 \ldots 3 \quad M_{A} e_{i}=L_{3}\left(e_{i} \otimes A^{-1} e_{3}\right) A^{-1} e_{3} .
$$

Then the unique minimizer $c_{0}=c\left(x^{\prime}, F_{2 \times 2}\right)$ in (3.1) is given by:

$$
A^{-1} c_{0}=-M_{A}^{-1} L_{3}\left(A^{-1} F_{2 \times 2}^{*} A^{-1}\right) A^{-1} e_{3} .
$$

Consequently:

$$
\mathcal{Q}_{2}\left(F_{2 \times 2}\right)=\mathcal{Q}_{3}\left(A^{-1} F_{2 \times 2}^{*} A^{-1}\right)-\left\langle M_{A}^{-1} L_{3}\left(A^{-1} F_{2 \times 2}^{*} A^{-1}\right) A^{-1} e_{3}, L_{3}\left(A^{-1} F_{2 \times 2}^{*} A^{-1}\right) A^{-1} e_{3}\right\rangle,
$$

Proof. For $i: 1 . .3$ we have:

$$
\begin{aligned}
\frac{\mathrm{d}}{\mathrm{d} c_{i}} \mathcal{Q}_{3}\left(A^{-1}\left(F_{2 \times 2}^{*}+c \otimes e_{3}\right) A^{-1}\right) & =2\left\langle L_{3}\left(A^{-1}\left(F_{2 \times 2}^{*}+c \otimes e_{3}\right) A^{-1}\right): A^{-1} e_{i} \otimes A^{-1} e_{3}\right\rangle \\
& =2\left\langle A^{-1} L_{3}\left(A^{-1}\left(F_{2 \times 2}^{*}+c \otimes e_{3}\right) A^{-1}\right) A^{-1}: e_{i} \otimes e_{3}\right\rangle .
\end{aligned}
$$

Therefore, at the miminizer $c_{0}$ we have:

$$
\begin{aligned}
\nabla_{c} \mathcal{Q}_{3}\left(A^{-1}\left(F_{2 \times 2}^{*}+c_{0} \otimes e_{3}\right) A^{-1}\right) & =2 A^{-1} L_{3}\left(A^{-1}\left(F_{2 \times 2}^{*}+c_{0} \otimes e_{3}\right) A^{-1}\right) A^{-1} e_{3} \\
& =2 A^{-1} L_{3}\left(A^{-1} F_{2 \times 2}^{*} A^{-1}+A^{-1} c_{0} \otimes A^{-1} e_{3}\right) A^{-1} e_{3}=0,
\end{aligned}
$$

which is equivalent to:

$$
-L_{3}\left(A^{-1} F_{2 \times 2}^{*} A^{-1}\right) A^{-1} e_{3}=L_{3}\left(A^{-1} c_{0} \otimes A^{-1} e_{3}\right) A^{-1} e_{3}=M_{A} A^{-1} c_{0},
$$


and consequently to (4.1). Then:

$$
\begin{aligned}
& \mathcal{Q}_{2}\left(F_{2 \times 2}\right)=\mathcal{Q}_{3}\left(A^{-1} F_{2 \times 2}^{*} A^{-1}+A^{-1} c_{0} \otimes A^{-1} e_{3}\right) \\
& \quad=\left\langle L_{3}\left(A^{-1} F_{2 \times 2}^{*} A^{-1}\right)+L_{3}\left(A^{-1} c_{0} \otimes A^{-1} e_{3}\right): A^{-1} F_{2 \times 2}^{*} A^{-1}\right\rangle \\
& \quad=\left\langle L_{3}\left(A^{-1} F_{2 \times 2}^{*} A^{-1}\right): A^{-1} F_{2 \times 2}^{*} A^{-1}+A^{-1} c_{0} \otimes A^{-1} e_{3}\right\rangle \\
& \quad=\mathcal{Q}_{3}\left(A^{-1} F_{2 \times 2}^{*} A^{-1}\right)-\left\langle L_{3}\left(A^{-1} F_{2 \times 2}^{*} A^{-1}\right): M_{A}^{-1} L_{3}\left(A^{-1} F_{2 \times 2}^{*} A^{-1}\right)\left(A^{-1} e_{3} \otimes A^{-1} e_{3}\right)\right\rangle,
\end{aligned}
$$

which proves (4.2).

We now assume that the energy density $W$ is isotropic, i.e.:

$$
\forall F \in \mathbb{R}^{3 \times 3} \quad \forall R \in S O(3) \quad W(R F)=W(F) .
$$

It is known [8] (see also [6] and Appendix A in [1]) that $\mathcal{Q}_{3}$ is then given in terms of the Lamé coefficients $\lambda, \mu$ :

$$
\mathcal{Q}_{3}(F)=\mu|\operatorname{sym} F|^{2}+\lambda|\operatorname{tr} F|^{2},
$$

and so we also have:

$$
L_{3}(F)=\mu \operatorname{sym} F+\lambda(\operatorname{tr} F) \operatorname{Id} .
$$

Lemma 4.2. Assume that $W$ is isotropic, so that (4.4) holds. Then:

$$
M_{A}=\frac{\mu}{2}\left|A^{-1} e_{3}\right|^{2} \operatorname{Id}+\left(\lambda+\frac{\mu}{2}\right)\left(A^{-1} e_{3} \otimes A^{-1} e_{3}\right)
$$

and, denoting $D=A^{-1} F_{2 \times 2}^{*} A^{-1}$ and $d=A^{-1} e_{3}$, we have:

$$
\forall F_{2 \times 2} \in \mathbb{R}_{\text {sym }}^{2 \times 2} \quad \mathcal{Q}_{2}\left(x^{\prime}, F_{2 \times 2}\right)=\mu\left(|D|^{2}-2 \frac{|D d|^{2}}{|d|^{2}}+\frac{\langle D d, d\rangle^{2}}{|d|^{4}}\right)+\frac{\lambda \mu}{\lambda+\mu}\left(\operatorname{tr} D-\frac{\langle D d, d\rangle}{|d|^{2}}\right)^{2} .
$$

Proof. By (4.5), we obtain:

$$
M_{A} e_{i}=L_{3}\left(e_{i} \otimes d\right) d=\left(\lambda+\frac{\mu}{2}\right)\left\langle d, e_{i}\right\rangle d+\frac{\mu}{2}|d|^{2} e_{i}
$$

which gives (4.6). It is easy to check directly the following general formula:

$$
(\alpha \operatorname{Id}+a \otimes b)^{-1}=\frac{1}{\alpha} \operatorname{Id}-\frac{1}{\alpha(\alpha+\langle a, b\rangle)} a \otimes b .
$$

Applying it to $\alpha=\frac{\mu}{2}|d|^{2}$ and $a=\left(\lambda+\frac{\mu}{2}\right) d$ and $b=d$, we get:

$$
M_{A}^{-1}=\frac{2}{\mu} \frac{1}{|d|^{2}} \operatorname{Id}-\frac{2 \lambda+\mu}{\mu(\lambda+\mu)} \frac{1}{|d|^{4}}(d \otimes d) .
$$

Therefore:

$$
\left\langle M_{A}^{-1} L_{3}(D) d, L_{3}(D) d\right\rangle=\frac{\lambda^{2}}{\lambda+\mu}(\operatorname{tr} D)^{2}+2 \frac{\lambda \mu}{\lambda+\mu}(\operatorname{tr} D) \frac{\langle D d, d\rangle}{|d|^{2}}+2 \mu \frac{|D d|^{2}}{|d|^{2}}-\frac{(2 \lambda+\mu) \mu}{\lambda+\mu} \frac{\langle D d, d\rangle^{2}}{|d|^{4}} .
$$

Concluding:

$$
\mathcal{Q}_{2}\left(x^{\prime}, F_{2 \times 2}\right)=\frac{\lambda \mu}{\lambda+\mu}(\operatorname{tr} D)^{2}+\mu|D|^{2}-2 \frac{\lambda \mu}{\lambda+\mu}(\operatorname{tr} D) \frac{\langle D d, d\rangle}{|d|^{2}}-2 \mu \frac{|D d|^{2}}{|d|^{2}}+\frac{(2 \lambda+\mu) \mu}{\lambda+\mu} \frac{\langle D d, d\rangle^{2}}{|d|^{4}}
$$

which yields (4.7). 
Theorem 4.3. Assume that $W$ is isotropic, so that (4.4) holds. Then:

$$
\begin{aligned}
\forall F_{2 \times 2} \in \mathbb{R}_{\text {sym }}^{2 \times 2} \quad & \mathcal{Q}_{2}\left(x^{\prime}, F_{2 \times 2}\right)=\mathcal{Q}_{2, i s o}^{0}\left({\sqrt{G_{2 \times 2}}}^{-1} F_{2 \times 2}{\sqrt{G_{2 \times 2}}}^{-1}\right) \\
& =\mu\left|{\sqrt{G_{2 \times 2}}-1}^{-1} F_{2 \times 2}{\sqrt{G_{2 \times 2}}}^{-1}\right|^{2}+\frac{\lambda \mu}{\lambda+\mu}\left|\operatorname{tr}\left({\sqrt{G_{2 \times 2}}-1}^{-1} F_{2 \times 2}{\sqrt{G_{2 \times 2}}}^{-1}\right)\right|^{2}
\end{aligned}
$$

Proof. Given $v \in \mathbb{R}^{3}$, we denote $v_{\text {tan }}=\left(v_{1}, v_{2}\right)^{T} \in \mathbb{R}^{2}$. As in the proof of Theorem 5.3, given $F \in \mathbb{R}^{3 \times 3}$, by $F_{\text {tan }} \in \mathbb{R}^{2 \times 2}$ we denote the principal $2 \times 2$ minor of $F$, and we let $F_{\text {cross }}=\left(F e_{3}\right)_{\tan }=$ $\left(F_{13}, F_{23}\right)^{T} \in \mathbb{R}^{2}$. We now use the notation of Lemma 4.2 and identify the terms in (4.7). Call $P=G^{-1}$. Then:

$$
\begin{aligned}
|D|^{2} & =\left\langle P F_{2 \times 2}^{*} P: F_{2 \times 2}^{*}\right\rangle=\left\langle\left(P F_{2 \times 2}^{*} P\right)_{\text {tan }}: F_{2 \times 2}\right\rangle=\left\langle P_{\text {tan }} F_{2 \times 2} P_{\text {tan }}: F_{2 \times 2}\right\rangle \\
|D d|^{2} & =\left\langle P F_{2 \times 2}^{*} P e_{3}, F_{2 \times 2}^{*} P e_{3}\right\rangle=\left\langle\left(P F_{2 \times 2}^{*} P e_{3}\right)_{\text {tan }}, F_{2 \times 2} P_{\text {cross }}\right\rangle=\left\langle P_{\text {tan }} F_{2 \times 2} P_{\text {cross }}, F_{2 \times 2} P_{\text {cross }}\right\rangle \\
\langle D d, d\rangle & =\left\langle P F_{2 \times 2}^{*} P e_{3}, e_{3}\right\rangle=\left\langle F P_{\text {cross }}, P_{\text {cross }}\right\rangle \\
|d|^{2} & =\left\langle P e_{3}, e_{3}\right\rangle=P_{33} \\
\operatorname{tr} D & =\operatorname{tr}\left(P F_{2 \times 2}^{*}\right)=\operatorname{tr}\left(P_{\text {tan }} F_{2 \times 2}\right) .
\end{aligned}
$$

Hence, (4.7) becomes:

$$
\begin{aligned}
\mathcal{Q}_{2}\left(x^{\prime}, F_{2 \times 2}\right)= & \mu\left(\left\langle P_{\text {tan }} F_{2 \times 2} P_{\text {tan }}: F_{2 \times 2}\right\rangle-2 \frac{\left\langle P_{\text {tan }} F_{2 \times 2} P_{\text {cross }}, F_{2 \times 2} P_{\text {cross }}\right\rangle}{P_{33}}+\frac{\left\langle F P_{\text {cross }}, P_{\text {cross }}\right\rangle^{2}}{\left(P_{33}\right)^{2}}\right) \\
& +\frac{\lambda \mu}{\lambda+\mu}\left(\operatorname{tr}\left(P_{\text {tan }} F_{2 \times 2}\right)-\frac{\left\langle F P_{\text {cross }}, P_{\text {cross }}\right\rangle}{P_{33}}\right)^{2} \cdot
\end{aligned}
$$

We now identify the terms in the right hand side of (4.8), using the formulas (5.9) and (5.10):

$$
\begin{aligned}
& \left|{\sqrt{G_{\text {tan }}}-1}^{-1} F_{2 \times 2} \sqrt{G_{\text {tan }}}-1\right|^{2}=\left\langle\left(G_{\text {tan }}\right)^{-1} F_{2 \times 2}\left(G_{\text {tan }}\right)^{-1}: F_{2 \times 2}\right\rangle \\
& =\left\langle\left(P_{\text {tan }}-\frac{1}{P_{33}} P_{\text {cross }} \otimes P_{\text {cross }}\right) F_{2 \times 2}\left(P_{\text {tan }}-\frac{1}{P_{33}} P_{\text {cross }} \otimes P_{\text {cross }}\right): F_{2 \times 2}\right\rangle \\
& =\left\langle P_{\text {tan }} F_{2 \times 2} P_{\text {tan }}: F_{2 \times 2}\right\rangle-\frac{2}{P_{33}}\left\langle\left(P_{\text {cross }} \otimes P_{\text {cross }}\right) F P_{\text {tan }}: F_{2 \times 2}\right\rangle \\
& \quad+\frac{1}{\left(P_{33}\right)^{2}}\left\langle\left(P_{\text {cross }} \otimes P_{\text {cross }}\right) F\left(P_{\text {cross }} \otimes P_{\text {cross }}\right): F\right\rangle \\
& =\left\langle P_{\text {tan }} F_{2 \times 2} P_{\text {tan }}: F_{2 \times 2}\right\rangle-2 \frac{\left\langle P_{\text {tan }} F_{2 \times 2} P_{\text {cross }}, F_{2 \times 2} P_{\text {cross }}\right\rangle}{P_{33}}+\frac{\left\langle F P_{\text {cross }}, P_{\text {cross }}\right\rangle^{2}}{\left(P_{33}\right)^{2}} \\
& \operatorname{tr}\left(\sqrt{G_{\text {tan }}}-1 F_{2 \times 2} \sqrt{G_{\text {tan }}}-1\right)=\operatorname{tr}\left(\left(G_{\text {tan }}\right)^{-1} F_{2 \times 2}\right)=\operatorname{tr}\left(\left(P_{\text {tan }}-\frac{1}{P_{33}} P_{\text {cross }} \otimes P_{\text {cross }}\right) F_{2 \times 2}\right) \\
& =\operatorname{tr}\left(P_{\text {tan }} F_{2 \times 2}\right)-\frac{1}{P_{33}}\left\langle F P_{\text {cross }}, P_{\text {cross }}\right\rangle .
\end{aligned}
$$

The equality in (4.8) follows directly by (4.9). 
Remark 4.4. When $G=G_{2 \times 2}^{*}+e_{3} \otimes e_{3}$ then $d=e_{3}$ and $D d=D e_{3}=0$, so (4.7) directly becomes:

$$
\mathcal{Q}_{2}\left(x^{\prime}, F_{2 \times 2}\right)=\mu|D|^{2}+\frac{\lambda \mu}{\lambda+\mu}|\operatorname{tr} D|^{2},
$$

which is consistent with (2.6).

Remark 4.5. Call $C\left(x^{\prime}\right)=G^{-1}\left(x^{\prime}\right) F_{2 \times 2}^{*}$ and note that:

$$
\begin{aligned}
& \operatorname{tr} D=\operatorname{tr} C, \quad|D|^{2}=\operatorname{tr}\left(C^{2}\right) \\
& |D d|^{2}=\left\langle C^{2} G^{-1} e_{3}, e_{3}\right\rangle, \quad|d|^{2}=\left\langle G^{-1} e_{3}, e_{3}\right\rangle, \quad\langle D d, d\rangle=\left\langle C G^{-1} e_{3}, e_{3}\right\rangle
\end{aligned}
$$

Consequently, (4.7) can also be equivalently written as:

$$
\mathcal{Q}_{2}\left(x^{\prime}, F_{2 \times 2}\right)=\mu\left(\operatorname{tr}\left(C^{2}\right)-2 \frac{\left\langle C^{2} G^{-1} e_{3}, e_{3}\right\rangle}{\left\langle G^{-1} e_{3}, e_{3}\right\rangle}+\frac{\left\langle C G^{-1} e_{3}, e_{3}\right\rangle^{2}}{\left\langle G^{-1} e_{3}, e_{3}\right\rangle^{2}}\right)+\frac{\lambda \mu}{\lambda+\mu}\left(\operatorname{tr} C-\frac{\left\langle C G^{-1} e_{3}, e_{3}\right\rangle}{\left\langle G^{-1} e_{3}, e_{3}\right\rangle}\right)^{2} .
$$

\section{Part B: Other limits}

\section{A CharaCterization of Bending: the 3D ENERGy SCALing AT Minimizers}

In this section we deduce the following property, complementary to Corollary 3.2.

Theorem 5.1. The non-vanishing of the three Riemann curvatures of the metric $G$ :

$$
\exists x \in \Omega \quad\left(\left|R_{112}^{3}\right|+\left|R_{221}^{3}\right|+\left|R_{1212}\right|\right)(x) \neq 0
$$

is equivalent to the lower bound on the energy scaling at minimizers:

$$
\exists c>0 \quad \inf _{u \in W^{1,2}\left(\Omega^{h}, \mathbb{R}^{3}\right)} E^{h}(u) \geq c h^{2} .
$$

Recall that the Riemann curvature tensor $R_{\ldots}$ and its covariant version $R \ldots$ are given by:

$$
\begin{aligned}
& R_{i j k}^{s}=\partial_{j} \Gamma_{i k}^{s}-\partial_{k} \Gamma_{i j}^{s}+\sum_{m=1}^{3} \Gamma_{j m}^{s} \Gamma_{i k}^{m}-\sum_{m=1}^{3} \Gamma_{k m}^{s} \Gamma_{i j}^{m} \\
& R_{s i j k}=\sum_{m=1}^{3} G_{s m} R_{i j k}^{m},
\end{aligned}
$$

while the Christoffel symbols are:

$$
\Gamma_{k l}^{i}=\frac{1}{2} \sum_{m=1}^{3} G^{i m}\left(\partial_{l} G_{m k}+\partial_{k} G_{m l}-\partial_{m} G_{k l}\right) .
$$

Remark 5.2. In [13] we proved for the metric $G$ having a $2 \mathrm{~d}$ structure $G=\left(G_{2 \times 2}\right)^{*}+e_{3} \otimes e_{3}$, that condition (5.2) is equivalent to the nonimmersability of $G$, i.e. nonvanishing of its full Riemann curvature tensor $R$. The reason for this seemingly more restricitve result is that for such $G$, its flatness is equivalent to the vanishing of the Gaussian curvature of $G_{2 \times 2}$, i.e. the $2 \mathrm{~d}$ flatness of the midplate metric $G_{2 \times 2}$. In fact, any isometric immersion of $G$ induces a flat isometric immersion of $G_{2 \times 2}$ whose second fundamental form $\Pi=0$ trivially satisfies the condition (5.5) below. We see that in the general case the curvatures that converge to the reduced $2 \mathrm{~d}$ energy $\mathcal{I}_{G}$ at the scaling $h^{2}$ are those listed in (5.1), rather than all the curvatures which naturally contribute towards residual 3 d energy $E^{h}$. 
The proof of Theorem 5.1 will follow directly from the next two theorems, which we present separately for their independent interest.

Theorem 5.3. The following conditions are equivalent:

(i) The energy functional (2.5) satisfies:

$$
\min \mathcal{I}_{G}=0,
$$

where the minimum is taken over isometric immersions of $G_{2 \times 2}$ in $\mathbb{R}^{3}$ of regularity $W^{2,2}$.

(ii) There exists a $W^{2,2}$ isometric immersion $y: \Omega \rightarrow \mathbb{R}^{3}$ of $G_{2 \times 2}$ such that:

$$
\operatorname{sym}\left((\nabla y)^{T} \nabla \vec{b}\right)=0 \quad \text { a.e. in } \Omega \text {. }
$$

where $\vec{b}: \Omega \rightarrow \mathbb{R}^{3}$ is uniquely defined by:

$$
\operatorname{det} Q>0 \quad \text { and } Q^{T} Q=G \text {, where: } Q e_{1}=\partial_{1} y, Q e_{2}=\partial_{2} y, Q e_{3}=\vec{b} .
$$

(iii) There exists a $W^{2,2}$ isometric immersion of $G_{2 \times 2}$ in $\mathbb{R}^{3}$, whose second fundamental form $\Pi$ is given by the Christoffel symbols of $G$ :

$$
\Pi_{11}=-\frac{1}{\sqrt{G^{33}}} \Gamma_{11}^{3}, \quad \Pi_{22}=-\frac{1}{\sqrt{G^{33}}} \Gamma_{22}^{3}, \quad \Pi_{12}=-\frac{1}{\sqrt{G^{33}}} \Gamma_{12}^{3} .
$$

Corollary 5.4. The unique (up to rigid motions) minimizing immersion in (5.3) is the immersion $y$ satisfying (5.4) and (5.5). This immersion is automatically smooth (up to the boundary of $\Omega$ ).

Proof. Let $y \in W^{2,2}\left(\Omega, \mathbb{R}^{3}\right)$ satisfy (2.2) and $\mathcal{I}_{G}(y)=0$. Denoting by $\vec{N} \in W^{1,2}\left(\Omega, \mathbb{R}^{3}\right)$ the unit normal to the surface $y(\Omega)$, we have $[9]$ :

$$
\partial_{i j} y=\sum_{m=1}^{2} \gamma_{i j}^{m} \partial_{m} y-\Pi_{i j} \vec{N} .
$$

Since $G_{2 \times 2}$ is smooth, then its Christoffel symbols $\gamma_{i j}^{m}$ are smooth, and also the coefficients in $\Pi_{i j}$ are smooth according to Theorem 5.3. Smoothness of $y$ follows then by a bootstrap argument.

Finally, uniqueness of isometric immersion with a prescribed second fundamental form, completes the proof.

Theorem 5.5. Conditions in Theorem 5.3 are further equivalent to the vanishing of the following three Riemann curvatures of $G$ :

$$
R_{112}^{3}=R_{221}^{3}=R_{1212}=0 .
$$

We now give proofs of Theorem 5.3 and Theorem 5.5

\section{Proof of Theorem 5.3.}

1. Condition (i) holds when there exists $y \in W^{2,2}\left(\Omega, \mathbb{R}^{3}\right)$ satisfying (2.2) and:

$$
\mathcal{I}_{G}(y)=0 .
$$

The equality (5.7) is clearly equivalent to: $\mathcal{Q}_{2}\left(x^{\prime},(\nabla y)^{T} \nabla \vec{b}\right)=0$ holding for a.e. $x^{\prime} \in \Omega$. Since $\mathcal{Q}_{3}(F)=0$ iff $F \in \mathbb{R}^{3 \times 3}$ is skew-symmetric, it follows that (5.7) is further equivalent to (5.4), hence proving (ii). 
2. Recall that the matrix field $Q$ in Theorem 2.1 (ii), whose columns are given by vectors $\partial_{1} y, \partial_{2} y$ and $\vec{b}$, satisfies $Q^{T} Q=G$. Hence, (5.7) becomes:

$$
\begin{aligned}
& 0=\left\langle\partial_{1} y, \partial_{1} \vec{b}\right\rangle=\partial_{1}\left\langle\partial_{1} y, \vec{b}\right\rangle-\left\langle\partial_{11} y, \vec{b}\right\rangle=\partial_{1} G_{13}-\left\langle\partial_{11} y, \vec{b}\right\rangle \\
& 0=\left\langle\partial_{2} y, \partial_{2} \vec{b}\right\rangle=\partial_{2}\left\langle\partial_{2} y, \vec{b}\right\rangle-\left\langle\partial_{22} y, \vec{b}\right\rangle=\partial_{2} G_{23}-\left\langle\partial_{22} y, \vec{b}\right\rangle \\
& 0=\left\langle\partial_{1} y, \partial_{2} \vec{b}\right\rangle+\left\langle\partial_{2} y, \partial_{1} \vec{b}\right\rangle=\partial_{1} G_{23}+\partial_{2} G_{13}-2\left\langle\partial_{12} y, \vec{b}\right\rangle .
\end{aligned}
$$

Given $F \in \mathbb{R}^{3 \times 3}$, by $F_{\text {tan }} \in \mathbb{R}^{2 \times 2}$ let us denote the principal $2 \times 2$ minor of $F$, and we let $F_{\text {cross }}=\left(F_{13}, F_{23}\right)^{T} \in \mathbb{R}^{2}$. Call $P=G^{-1}$ Then:

$$
G_{\text {tan }} P_{\text {tan }}+G_{\text {cross }} \otimes P_{\text {cross }}=\mathrm{Id}_{2}, \quad G_{\text {tan }} P_{\text {cross }}+P_{33} G_{\text {cross }}=0,
$$

and so consequently:

$$
\left(G_{\text {tan }}\right)^{-1}=P_{\text {tan }}+\left(G_{\text {tan }}\right)^{-1} G_{\text {cross }} \otimes P_{\text {cross }}=P_{\text {tan }}-\frac{1}{P_{33}} P_{\text {cross }} \otimes P_{\text {cross }} .
$$

We therefore obtain:

$$
G^{33}=P_{33}=\frac{\operatorname{det} G_{2 \times 2}}{\operatorname{det} G}, \quad\left(G_{\text {tan }}\right)^{-1} G_{\text {cross }}=-\frac{1}{P_{33}} P_{\text {cross }}=-\frac{1}{G^{33}}\left(G^{13}, G^{23}\right)^{T},
$$

where as the standard notation is used: $\left[G^{i j}\right]_{i, j: 1 . .3}=G^{-1}=P$. It follows by (2.3) that:

$$
\vec{b}=-\frac{1}{G^{33}}\left(G^{13} \partial_{1} y+G^{23} \partial_{2} y\right)+\frac{1}{\sqrt{G^{33}}} \vec{N},
$$

and hence (5.8) becomes:

$$
\begin{aligned}
\partial_{1} G_{13} & =-\frac{1}{G^{33}}\left(G^{13}\left\langle\partial_{11} y, \partial_{1} y\right\rangle+G^{23}\left\langle\partial_{11} y, \partial_{2} y\right\rangle\right)-\frac{1}{\sqrt{G^{33}}} \Pi_{11} \\
\partial_{2} G_{23} & =-\frac{1}{G^{33}}\left(G^{13}\left\langle\partial_{22} y, \partial_{1} y\right\rangle+G^{23}\left\langle\partial_{22} y, \partial_{2} y\right\rangle\right)-\frac{1}{\sqrt{G^{33}}} \Pi_{22} \\
\frac{1}{2}\left(\partial_{1} G_{23}+\partial_{2} G_{13}\right) & =-\frac{1}{G^{33}}\left(G^{13}\left\langle\partial_{12} y, \partial_{1} y\right\rangle+G^{23}\left\langle\partial_{12} y, \partial_{2} y\right\rangle\right)-\frac{1}{\sqrt{G^{33}}} \Pi_{12},
\end{aligned}
$$

where we used the fact that the coefficients of the second fundamental form $\Pi$ of the surface $y(\Omega)$ satisfy: $\Pi_{i j}=\left\langle\partial_{i} y, \partial_{j} \vec{N}\right\rangle=-\left\langle\partial_{i j} y, \vec{N}\right\rangle$ for $i, j: 1 . .2$.

Also, note that $\partial_{i} G=2 \operatorname{sym}\left(\left(\partial_{i} Q\right)^{T} Q\right)$ for $i=1,2$, from where we deduce:

$$
\begin{aligned}
\left\langle\partial_{11} y, \partial_{1} y\right\rangle & =\frac{1}{2} \partial_{1} G_{11}, & \left\langle\partial_{22} y, \partial_{2} y\right\rangle & =\frac{1}{2} \partial_{2} G_{22}, \\
\left\langle\partial_{12} y, \partial_{1} y\right\rangle & =\frac{1}{2} \partial_{2} G_{11}, & \left\langle\partial_{12} y, \partial_{2} y\right\rangle & =\frac{1}{2} \partial_{1} G_{22}, \\
\left\langle\partial_{11} y, \partial_{2} y\right\rangle=\partial_{1} G_{12} & -\frac{1}{2} \partial_{2} G_{11}, & \left\langle\partial_{22} y, \partial_{1} y\right\rangle & =\partial_{2} G_{12}-\frac{1}{2} \partial_{1} G_{22} .
\end{aligned}
$$

3. We now want to rewrite the equations in (5.11) and the formulas (5.12) using the Christoffel symbols $\Gamma_{i j}^{m}, i, j, m=1 . .3$ of the metric $G$. Recall that, since the Levi-Civita connection is metric-compatible, we have:

$$
\partial_{i} G_{j k}=\sum_{m=1}^{3} G_{m k} \Gamma_{i j}^{m}+\sum_{m=1}^{3} G_{m j} \Gamma_{i k}^{m},
$$




$$
\partial_{i} G^{j k}=-\sum_{m=1}^{3} G^{m k} \Gamma_{m i}^{j}-\sum_{m=1}^{3} G^{m j} \Gamma_{m i}^{k} .
$$

Since $\partial_{3} G=0$, it follows that:

$$
\sum_{m=1}^{3} G_{m 1} \Gamma_{13}^{m}=0, \quad \sum_{m=1}^{3} G_{m 2} \Gamma_{23}^{m}=0 \quad \sum_{m=1}^{3}\left(G_{m 2} \Gamma_{13}^{m}+G_{m 1} \Gamma_{23}^{m}\right)=0 .
$$

Therefore and in view of (5.13), (5.12) become:

$$
\begin{aligned}
\left\langle\partial_{11} y, \partial_{1} y\right\rangle & =\sum_{m=1}^{3} G_{m 1} \Gamma_{11}^{m}, & \left\langle\partial_{22} y, \partial_{2} y\right\rangle & =\sum_{m=1}^{3} G_{m 2} \Gamma_{22}^{m}, \\
\left\langle\partial_{12} y, \partial_{1} y\right\rangle & =\sum_{m=1}^{3} G_{m 1} \Gamma_{12}^{m}, & \left\langle\partial_{12} y, \partial_{2} y\right\rangle & =\sum_{m=1}^{3} G_{m 2} \Gamma_{12}^{m}, \\
\left\langle\partial_{11} y, \partial_{2} y\right\rangle & =\sum_{m=1}^{3} G_{m 2} \Gamma_{11}^{m}, & \left\langle\partial_{22} y, \partial_{1} y\right\rangle & =\sum_{m=1}^{3} G_{m 1} \Gamma_{22}^{m} .
\end{aligned}
$$

By (5.11), (5.15) and (5.13) we now obtain:

$$
\begin{aligned}
& \Pi_{11}=-\frac{1}{\sqrt{G^{33}}} \sum_{m=1}^{3}\left(G^{13} G_{m 1}+G^{23} G_{m 2}\right) \Gamma_{11}^{m}-\sqrt{G^{33}} \sum_{m=1}^{3} G_{m 3} \Gamma_{11}^{m}, \\
& \Pi_{22}=-\frac{1}{\sqrt{G^{33}}} \sum_{m=1}^{3}\left(G^{13} G_{m 1}+G^{23} G_{m 2}\right) \Gamma_{22}^{m}-\sqrt{G^{33}} \sum_{m=1}^{3} G_{m 3} \Gamma_{22}^{m}, \\
& \Pi_{12}=-\frac{1}{\sqrt{G^{33}}} \sum_{m=1}^{3}\left(G^{13} G_{m 1}+G^{23} G_{m 2}\right) \Gamma_{12}^{m}-\sqrt{G^{33}} \sum_{m=1}^{3} G_{m 3} \Gamma_{12}^{m} .
\end{aligned}
$$

Since $\sum_{i=1}^{3} G^{i 3} G_{m i}=\delta_{m 3}$, we conclude (5.5) and note that it is equivalent to (5.7).

\section{Proof of Theorem 5.5 .}

Clearly, Theorem 5.3 (iii) is equivalent (see [9] for details) to the satisfaction of the CodazziMainardi equations for the $2 \mathrm{~d}$ metric $G_{2 \times 2}$ and the second fundamental form $\Pi$ :

$$
\begin{aligned}
& \partial_{2}\left(\frac{1}{\sqrt{G^{33}}} \Gamma_{11}^{3}\right)-\partial_{1}\left(\frac{1}{\sqrt{G^{33}}} \Gamma_{12}^{3}\right)=\frac{1}{\sqrt{G^{33}}}\left(\sum_{m=1}^{2} \Gamma_{1 m}^{3} \gamma_{12}^{m}-\sum_{m=1}^{2} \Gamma_{2 m}^{3} \gamma_{11}^{m}\right) \\
& \partial_{2}\left(\frac{1}{\sqrt{G^{33}}} \Gamma_{12}^{3}\right)-\partial_{1}\left(\frac{1}{\sqrt{G^{33}}} \Gamma_{22}^{3}\right)=\frac{1}{\sqrt{G^{33}}}\left(\sum_{m=1}^{2} \Gamma_{1 m}^{3} \gamma_{22}^{m}-\sum_{m=1}^{2} \Gamma_{2 m}^{3} \gamma_{12}^{m}\right)
\end{aligned}
$$

together with the Gauss equation:

$$
\Gamma_{11}^{3} \Gamma_{22}^{3}-\left(\Gamma_{12}^{3}\right)^{2}=G^{33} \kappa \operatorname{det} G_{2 \times 2} .
$$

Above, by $\gamma_{i j}^{k}$ we denote the Christoffel symbols of the metric $G_{2 \times 2}$, while $\kappa=\kappa\left(G_{2 \times 2}\right)$ is the Gaussian curvature of $G_{2 \times 2}$. We now prove that (5.16) and (5.17) are equivalent to (5.6). 
1. We first relate the Christoffel symbols $\gamma_{k l}^{s}$ with $\Gamma_{k l}^{s}$. By (15.9), the inverse matrix $\left[g^{i j}\right]_{i, j=1 . .2}=$ $\left[\left(G_{2 \times 2}\right)^{-1}\right]_{i j}$ is given by: $g^{i j}=G^{i j}-\frac{1}{G^{33}} G^{3 i} G^{3 j}$. Hence:

$$
\begin{aligned}
\gamma_{k l}^{s} & =\frac{1}{2} \sum_{m=1}^{2} g^{s m}\left(\partial_{l} G_{m k}+\partial_{k} G_{m l}-\partial_{m} G_{k l}\right) \\
& =\frac{1}{2} \sum_{m=1}^{2} G^{s m}\left(\partial_{l} G_{m k}+\partial_{k} G_{m l}-\partial_{m} G_{k l}\right)-\frac{1}{2} \frac{G^{3 s}}{G^{33}} \sum_{m=1}^{2} G^{3 m}\left(\partial_{l} G_{m k}+\partial_{k} G_{m l}-\partial_{m} G_{k l}\right) \\
& =\Gamma_{k l}^{s}-G^{3 s}\left(\partial_{l} G_{3 k}+\partial_{k} G_{3 l}\right)-\frac{G^{3 s}}{G^{33}}\left(\Gamma_{k l}^{3}-G^{33}\left(\partial_{l} G_{3 k}+\partial_{k} G_{3 l}\right)\right) \\
& =\Gamma_{k l}^{s}-\frac{G^{3 s}}{G^{33}} \Gamma_{k l}^{3} .
\end{aligned}
$$

Also, note that by (5.14), for $i=1,2$ we have:

$$
\sqrt{G^{33}} \partial_{i}\left(\frac{1}{\sqrt{G^{33}}}\right)=-\frac{1}{2} \frac{\partial_{i} G^{33}}{G^{33}}=\frac{1}{G^{33}} \sum_{m=1}^{3} G^{m 3} \Gamma_{m i}^{3} .
$$

2. The first equation in (5.16) now becomes:

$$
\begin{aligned}
\partial_{2} \Gamma_{11}^{3}-\partial_{1} \Gamma_{12}^{3}-\frac{1}{2} & \left(\frac{\partial_{2} G^{33}}{G^{33}} \Gamma_{11}^{3}-\frac{\partial_{1} G^{33}}{G^{33}} \Gamma_{12}^{3}\right) \\
& =\left(\sum_{m=1}^{2} \Gamma_{1 m}^{3} \Gamma_{12}^{m}-\sum_{m=1}^{2} \Gamma_{2 m}^{3} \Gamma_{11}^{m}\right)+\frac{G^{32}}{G^{33}}\left(\Gamma_{11}^{3} \Gamma_{22}^{3}-\left(\Gamma_{12}^{3}\right)^{2}\right)
\end{aligned}
$$

Therefore, in view of (5.19) we obtain:

$$
\begin{aligned}
R_{121}^{3} & =\partial_{2} \Gamma_{11}^{3}-\partial_{1} \Gamma_{12}^{3}+\sum_{m=1}^{3}\left(\Gamma_{2 m}^{3} \Gamma_{11}^{m}-\Gamma_{1 m}^{3} \Gamma_{12}^{m}\right) \\
& =\frac{1}{G^{33}}\left(G^{33}\left(\Gamma_{23}^{3} \Gamma_{11}^{3}-\Gamma_{13}^{3} \Gamma_{12}^{3}\right)+\frac{1}{2}\left(\partial_{2} G^{33} \Gamma_{11}^{3}-\partial_{1} G^{33} \Gamma_{12}^{3}\right)+G^{32}\left(\Gamma_{11}^{3} \Gamma_{22}^{3}-\left(\Gamma_{12}^{3}\right)^{2}\right)\right) \\
& =\frac{1}{G^{33}}\left(\sum_{m=1}^{2}\left(G^{m 3} \Gamma_{m 1}^{3} \Gamma_{12}^{3}-G^{m 3} \Gamma_{m 2}^{3} \Gamma_{11}^{3}\right)+G^{32}\left(\Gamma_{11}^{3} \Gamma_{22}^{3}-\left(\Gamma_{12}^{3}\right)^{2}\right)\right)
\end{aligned}
$$

which gives $R_{121}^{3}=0$ by direct inspection. Similarly, the second equation in (5.16) yields:

$$
\begin{aligned}
\partial_{2} \Gamma_{12}^{3}-\partial_{1} \Gamma_{22}^{3}-\frac{1}{2} & \left(\frac{\partial_{2} G^{33}}{G^{33}} \Gamma_{12}^{3}-\frac{\partial_{1} G^{33}}{G^{33}} \Gamma_{22}^{3}\right) \\
& =\left(\sum_{m=1}^{2} \Gamma_{1 m}^{3} \Gamma_{22}^{m}-\sum_{m=1}^{2} \Gamma_{2 m}^{3} \Gamma_{12}^{m}\right)+\frac{G^{31}}{G^{33}}\left(\Gamma_{11}^{3} \Gamma_{22}^{3}-\left(\Gamma_{12}^{3}\right)^{2}\right)
\end{aligned}
$$


Consequently, using (5.19) as before:

$$
\begin{aligned}
R_{221}^{3} & =\partial_{2} \Gamma_{12}^{3}-\partial_{1} \Gamma_{22}^{3}+\sum_{m=1}^{3}\left(\Gamma_{2 m}^{3} \Gamma_{12}^{m}-\Gamma_{1 m}^{3} \Gamma_{22}^{m}\right) \\
& =\frac{1}{G^{33}}\left(G^{33}\left(\Gamma_{23}^{3} \Gamma_{12}^{3}-\Gamma_{13}^{3} \Gamma_{22}^{3}\right)+\frac{1}{2}\left(\partial_{2} G^{33} \Gamma_{12}^{3}-\partial_{1} G^{33} \Gamma_{22}^{3}\right)-G^{31}\left(\Gamma_{11}^{3} \Gamma_{22}^{3}-\left(\Gamma_{12}^{3}\right)^{2}\right)\right) \\
& =\frac{1}{G^{33}}\left(\sum_{m=1}^{2}\left(G^{m 3} \Gamma_{m 1}^{3} \Gamma_{22}^{3}-G^{m 3} \Gamma_{m 2}^{3} \Gamma_{12}^{3}\right)-G^{31}\left(\Gamma_{11}^{3} \Gamma_{22}^{3}-\left(\Gamma_{12}^{3}\right)^{2}\right)\right)
\end{aligned}
$$

which implies $R_{221}^{3}=0$.

3. We now turn to proving equivalence of (5.17) with $R_{1212}=0$. Denoting $r_{i j k}^{s}$ and $r_{s i j k}$ the Riemann curvatures of the metric $G_{2 \times 2}$ (where $i, j, k, s=1 . .2$ ) we obtain:

$$
\kappa \operatorname{det} G_{2 \times 2}=r_{1212}=G_{11} r_{212}^{1}+G_{12} r_{212}^{2}
$$

Further, for $i=1,2$ we get by (5.18) and (5.14):

$$
\begin{aligned}
r_{212}^{i}= & \partial_{1}\left(\Gamma_{22}^{i}-\frac{G^{3 i}}{G^{33}} \Gamma_{22}^{3}\right)-\partial_{2}\left(\Gamma_{12}^{i}-\frac{G^{3 i}}{G^{33}} \Gamma_{12}^{3}\right) \\
& +\sum_{m=1}^{2}\left(\Gamma_{1 m}^{i}-\frac{G^{3 i}}{G^{33}} \Gamma_{1 m}^{3}\right)\left(\Gamma_{22}^{m}-\frac{G^{3 m}}{G^{33}} \Gamma_{22}^{3}\right)-\sum_{m=1}^{2}\left(\Gamma_{2 m}^{i}-\frac{G^{3 i}}{G^{33}} \Gamma_{2 m}^{3}\right)\left(\Gamma_{12}^{m}-\frac{G^{3 m}}{G^{33}} \Gamma_{12}^{3}\right) \\
= & R_{212}^{i}-\frac{G^{3 i}}{G^{33}}\left(\partial_{1} \Gamma_{22}^{3}-\partial_{2} \Gamma_{12}^{3}\right)+\left(\frac{G^{1 i}}{G^{33}}-\frac{G^{3 i} G^{31}}{\left(G^{33}\right)^{2}}\right)\left(\Gamma_{11}^{3} \Gamma_{22}^{3}-\left(\Gamma_{12}^{3}\right)^{2}\right) \\
& -\frac{G^{3 i}}{G^{33}} \sum_{m=1}^{3}\left(\Gamma_{1 m}^{3} \Gamma_{22}^{m}-\Gamma_{2 m}^{3} \Gamma_{12}^{m}\right) .
\end{aligned}
$$

Consequently, the Gauss equation (5.17) yields:

$$
\begin{aligned}
R_{1212}= & G_{11} R_{212}^{1}+G_{12} R_{212}^{2}+G_{13} R_{212}^{3} \\
= & \kappa \operatorname{det} G_{2 \times 2}+G_{11}\left(R_{212}^{1}-r_{212}^{1}\right)+G_{12}\left(R_{212}^{2}-r_{212}^{2}\right)+G_{13} R_{212}^{3} \\
= & G_{13} R_{212}^{3}+\frac{1}{G^{33}}\left(\Gamma_{11}^{3} \Gamma_{22}^{3}-\left(\Gamma_{12}^{3}\right)^{2}\right)-G_{13}\left(\partial_{1} \Gamma_{22}^{3}-\partial_{2} \Gamma_{12}^{3}\right) \\
& -\left(\frac{\left(1-G^{13} G_{13}\right)}{G^{33}}+\frac{G^{33} G_{13} G^{31}}{\left(G^{33}\right)^{2}}\right)\left(\Gamma_{11}^{3} \Gamma_{22}^{3}-\left(\Gamma_{12}^{3}\right)^{2}\right)-G_{31} \sum_{m=1}^{3}\left(\Gamma_{1 m}^{3} \Gamma_{22}^{m}-\Gamma_{2 m}^{3} \Gamma_{12}^{m}\right) \\
= & G_{13} R_{212}^{3}-G_{13}\left(\partial_{1} \Gamma_{22}^{3}-\partial_{2} \Gamma_{12}^{3}+\sum_{m=1}^{3}\left(\Gamma_{1 m}^{3} \Gamma_{22}^{m}-\Gamma_{2 m}^{3} \Gamma_{12}^{m}\right)\right)=0 .
\end{aligned}
$$

Note that we did not use the fact that $R_{212}^{3}=0$ in the above calculation. This completes the proof of Theorem 5.5.

\section{EXAMPLES}

Example 6.1. Let $\lambda: \bar{\Omega} \rightarrow \mathbb{R}$ be a smooth positive function and define:

$$
G\left(x^{\prime}, x_{3}\right)=G\left(x^{\prime}\right)=\operatorname{diag}\left(1,1, \lambda\left(x^{\prime}\right)\right) .
$$


Clearly, the $2 \mathrm{~d}$ metric $G_{2 \times 2}=\mathrm{Id}_{2}$ has an isometric immersion $y_{0}\left(x^{\prime}\right)=x^{\prime}$ with the second fundamental form $\Pi=0$. On the other hand:

$$
\forall i, j: 1 . .2 \quad \Gamma_{i j}^{3}=\frac{1}{2 \lambda}\left(\partial_{i} G_{3 j}+\partial_{j} G_{3 i}\right)=0,
$$

and we see that both conditions (i) and (ii) in Theorem 5.3 are satisfied, so that $\mathcal{I}_{G}\left(y_{0}\right)=0$.

We can further check directly that the only possibly non-zero Christoffel symbols are:

$$
\forall i: 1 . .2 \quad \Gamma_{33}^{i}=-\frac{1}{2} \partial_{i} \lambda, \quad \Gamma_{i 3}^{3}=\frac{1}{2 \lambda} \partial_{i} \lambda .
$$

In particular, it easily follows that: $R_{121}^{3}=R_{221}^{3}=R_{1212}=0$, which is consistent with Theorem 5.5. At the same time $G$ is, in general, non-immersable. To see this, recall that the Ricci curvatures are given by: $R_{i j}=\sum_{l=1}^{3}\left(\partial_{l} \Gamma_{i j}^{l}-\partial_{j} \Gamma_{i l}^{l}\right)+\sum_{l, m=1}^{3}\left(\Gamma_{i j}^{l} \Gamma_{l m}^{m}-\Gamma_{i l}^{m} \Gamma_{j m}^{l}\right)$, for $i, j=1 \ldots 3$. In the present case, we have:

$$
\begin{aligned}
& R_{11}=\frac{1}{4 \lambda^{2}}\left(\left(\partial_{1} \lambda\right)^{2}-2 \lambda\left(\partial_{11} \lambda\right)\right), \quad R_{12}=\frac{1}{4 \lambda^{2}}\left(\left(\partial_{1} \lambda\right)\left(\partial_{2} \lambda\right)-2 \lambda\left(\partial_{12} \lambda\right)\right), \quad R_{13}=R_{23}=0, \\
& R_{22}=\frac{1}{4 \lambda^{2}}\left(\left(\partial_{2} \lambda\right)^{2}-2 \lambda\left(\partial_{22} \lambda\right)\right), \quad R_{33}=-\frac{1}{4 \lambda}\left(\left(\partial_{1} \lambda\right)^{2}-2 \lambda\left(\partial_{11} \lambda\right)+\left(\partial_{2} \lambda\right)^{2}-2 \lambda\left(\partial_{22} \lambda\right)\right) .
\end{aligned}
$$

We hence see that $G$ is immersable if and only if:

$$
M:=\nabla^{2} \lambda-\frac{1}{2 \lambda} \nabla \lambda \otimes \nabla \lambda \equiv 0 \quad \text { in } \Omega .
$$

Let us now consider the scaling of the $3 \mathrm{~d}$ non-Euclidean energy studied in this paper:

$$
E^{h}\left(u^{h}\right)=\frac{1}{h} \int_{\Omega^{h}} W\left(\nabla u^{h} \sqrt{G}^{-1}\right) \mathrm{d} x, \quad \sqrt{G}^{-1}=\operatorname{diag}\left(1,1, \frac{1}{\sqrt{\lambda}}\right),
$$

at the following sequence of smooth deformations of $\Omega^{h}$ :

$$
u^{h}\left(x^{\prime}, x_{3}\right)=x^{\prime}+\left(-\frac{x_{3}^{2}}{4} \partial_{1} \lambda,-\frac{x_{3}^{2}}{4} \partial_{2} \lambda, \sqrt{\lambda} x_{3}\right)^{T} .
$$

We have: $\left(\left(\nabla u^{h}\right) \sqrt{G}^{-1}\right)_{2 \times 2}=\operatorname{Id}_{2}-\frac{x_{3}^{2}}{4} \nabla^{2} \lambda$, and:

$$
\left(\left(\nabla u^{h}\right) \sqrt{G}^{-1}\right) e_{3}=\left(-\frac{x_{3}}{2} \frac{\partial_{1} \lambda}{\sqrt{\lambda}},-\frac{x_{3}}{2} \frac{\partial_{2} \lambda}{\sqrt{\lambda}}, 1\right)^{T}, \quad\left(\left(\nabla u^{h}\right) \sqrt{G}^{-1}\right)^{T} e_{3}=\left(\frac{x_{3}}{2} \frac{\partial_{1} \lambda}{\sqrt{\lambda}}, \frac{x_{3}}{2} \frac{\partial_{2} \lambda}{\sqrt{\lambda}}, 1\right)^{T} .
$$

Recall that for every $F=\operatorname{Id}_{3}+\mathcal{A} \in \mathbb{R}^{3 \times 3}$ when $\mathcal{A}$ is sufficiently small, we have: $\operatorname{dist}(F, S O(3))=$ $\left|\sqrt{F^{T} F}-\mathrm{Id}\right|=\left|\sqrt{\mathrm{Id}+2 \operatorname{sym} \mathcal{A}+\mathcal{A}^{T} \mathcal{A}}-\mathrm{Id}\right|=\left|\operatorname{sym} \mathcal{A}+\frac{1}{2} \mathcal{A}^{T} \mathcal{A}+o\left(\left|\operatorname{sym} \mathcal{A}+\frac{1}{2} \mathcal{A}^{T} \mathcal{A}\right|\right)\right|$. Consequently:

$$
W\left(\left(\nabla u^{h}\right) \sqrt{G}^{-1}\right) \leq C \operatorname{dist}^{2}\left(\left(\nabla u^{h}\right) \sqrt{G}^{-1}, S O(3)\right) \leq C x_{3}^{4}
$$

and therefore:

$$
\inf E^{h} \leq E^{h}\left(u^{h}\right) \leq \frac{C}{h} \int_{-h / 2}^{h / 2} x_{3}^{4} \mathrm{~d} x_{3}=C h^{4},
$$

for any choice of $\lambda$ in (6.1). In section 7 we will show that this scaling is also optimal, provided that the condition (6.2) does not hold. 
Example 6.2. Let $\lambda: \bar{\Omega} \rightarrow \mathbb{R}$ be a smooth positive function and consider the metric:

$$
G\left(x^{\prime}, x_{3}\right)=G\left(x^{\prime}\right)=\lambda\left(x^{\prime}\right) \operatorname{Id}_{3} .
$$

One checks directly that $\Gamma_{k l}^{i}=\frac{1}{2 \lambda}\left(\delta_{i k} \partial_{l} \lambda+\delta_{i l} \partial_{k} \lambda-\delta_{k l} \partial_{i} \lambda\right)=\delta_{i k} \partial_{l} f+\delta_{i l} \partial_{k} f-\delta_{k l} \partial_{i} f$, where we denote $f=\frac{1}{2} \log \lambda$. We directly compute:

$$
R_{112}^{3}=R_{221}^{3}=0 \quad \text { and } \quad R_{1212}=-\frac{1}{2} \lambda \Delta(\log \lambda)=\lambda^{2} \kappa\left(\lambda \operatorname{Id}_{2}\right) .
$$

Therefore, condition (5.6) which is equivalent to $\min \mathcal{I}_{G}=0$ according to Theorem 5.5, holds if and only if:

$$
\Delta(\log \lambda)=0,
$$

or equivalently, when the 2 d metric $G_{2 \times 2}=\lambda \operatorname{Id}_{2}$ is flat (immersable in $\mathbb{R}^{2}$ ). Note also that since $\Gamma_{i j}^{3}=0$ for $i, j: 1 . .2$ then this is precisely the case when (5.5) of Theorem 5.3 is satisfied.

We now compute the Ricci curvature of $G$ using the conformal rescaling formula:

$$
\begin{aligned}
\operatorname{Ric}(G) & =\operatorname{Ric}\left(e^{2 f} \operatorname{Id}_{3}\right)=-\left(\nabla^{2} f-\nabla f \otimes \nabla f\right)-\left(\Delta f-|\nabla f|^{2}\right) \operatorname{Id}_{3} \\
& =\left(-2(\Delta f) \operatorname{Id}_{2}+\operatorname{cof}\left(\nabla^{2} f-\nabla f \otimes \nabla f\right)\right)^{*}-\left(\Delta f+|\nabla f|^{2}\right) e_{3} \otimes e_{3} .
\end{aligned}
$$

We observe that $G$ is immersable iff $\operatorname{Ric}(G)=0$, i.e. when $\nabla f=0$, which is equivalent to:

$$
\lambda \equiv \text { const. }
$$

Clearly (6.7) implies (6.6), but conversely: there exist nonimmersable metrics $G$ for which (6.6) holds i.e. for which the minimum of the residual energy $\mathcal{I}_{G}$ is 0 , and it is attained by the unique (up to rigid motions) smooth isometric immersion $y: \Omega \rightarrow \mathbb{R}^{2}$ of $\lambda \operatorname{Id}_{2}$.

As in Example 6.1, we now consider scaling of the 3 d energies $E^{h}$, assuming (6.6). Define:

$$
u^{h}\left(x^{\prime}, x_{3}\right)=y\left(x^{\prime}\right)^{*}+x_{3} \sqrt{\lambda} e_{3}-\frac{x_{3}^{2}}{4}\left((\nabla y)^{-1, T} \nabla \lambda\right)^{*} .
$$

We easily compute that $\left(\nabla u^{h}\left(x^{\prime}, x_{3}\right)\right)_{2 \times 2}=\nabla y+\mathcal{O}\left(x_{3}^{2}\right)$ and:

$$
\begin{aligned}
\left(\nabla u^{h}\left(x^{\prime}, x_{3}\right)\right) e_{3} & =\left(-\frac{x_{3}}{2}\left\langle(\nabla y)^{-1, T} \nabla \lambda, e_{1}\right\rangle,-\frac{x_{3}}{2}\left\langle(\nabla y)^{-1, T} \nabla \lambda, e_{2}\right\rangle, \sqrt{\lambda}\right)^{T}, \\
\left(\nabla u^{h}\left(x^{\prime}, x_{3}\right)\right)^{T} e_{3} & =\left(\frac{x_{3}}{2 \sqrt{\lambda}} \partial_{1} \lambda, \frac{x_{3}}{2 \sqrt{\lambda}} \partial_{2} \lambda, \sqrt{\lambda}\right)^{T} .
\end{aligned}
$$

Since $(\nabla y)^{T} \nabla y=\lambda \mathrm{Id}_{2}$ it follows that:

$$
\begin{aligned}
& \left(\left(\nabla u^{h}\right)^{T} \nabla u^{h}\right)_{2 \times 2}=\lambda \operatorname{Id}_{2}+\mathcal{O}\left(x_{3}^{2}\right) \\
\forall i=1 . .2 & \left(\left(\nabla u^{h}\right)^{T} \nabla u^{h}\right)_{3 i}=-\frac{x_{3}}{2}\left\langle\partial_{i} y,(\nabla y)^{-1, T} \nabla \lambda\right\rangle+\frac{x_{3}}{2} \partial_{i} \lambda+\mathcal{O}\left(x_{3}^{2}\right)=\mathcal{O}\left(x_{3}^{2}\right) \\
& \left(\left(\nabla u^{h}\right)^{T} \nabla u^{h}\right)_{33}=\lambda+\mathcal{O}\left(x_{3}^{2}\right) .
\end{aligned}
$$

Therefore it follows, by polar decomposition theorem:

$$
\begin{aligned}
W\left(\left(\nabla u^{h}\right) \sqrt{G}^{-1}\right) & \leq C \operatorname{dist}^{2}\left(\frac{1}{\sqrt{\lambda}} \nabla u^{h}, S O(3)\right) \leq C \operatorname{dist}^{2}\left(\sqrt{\frac{1}{\lambda}\left(\nabla u^{h}\right)^{T} \nabla u^{h}}, S O(3)\right) \\
& =C \operatorname{dist}^{2}\left(\sqrt{\operatorname{Id}_{3}+\mathcal{O}\left(x_{3}^{2}\right)}, S O(3)\right) \leq C x_{3}^{4},
\end{aligned}
$$


which again yields the scaling $h^{4}$, precisely as in (6.4).

Remark 6.3. A more general example of $G$ in the same spirit as above, is: $G\left(x^{\prime}\right)=G_{2 \times 2}^{*}+$ $\lambda\left(x^{\prime}\right) e_{3} \otimes e_{3}$ with $G_{2 \times 2}$ immersable in $\mathbb{R}^{2}$. Since $\Gamma_{i j}^{3}=0$ for $i, j=1 . .2$, we see that $\min \mathcal{I}_{G}=0$, in virtue of Theorem 5.3. On the other hand, one can check directly that taking smooth $y: \Omega \rightarrow \mathbb{R}^{2}$ such that $(\nabla y)^{T} \nabla y=G_{2 \times 2}$ and defining the $3 \mathrm{~d}$ deformations $u^{h}$ as in (6.8), it again follows: $W\left(\left(\nabla u^{h}\right) \sqrt{G}^{-1}\right) \leq C x_{3}^{4}$. Consequently, the same energy scaling as in (6.4) is valid here as well.

Example 6.4. (i). Let $\lambda_{1}, \lambda_{2}, \lambda_{3}: \bar{\Omega} \rightarrow \mathbb{R}$ be smooth functions such that $\lambda_{3}>\lambda_{1}^{2}+\lambda_{2}^{2}$. Define:

$$
G\left(x^{\prime}, x_{3}\right)=G\left(x^{\prime}\right)=\left[\begin{array}{ccc}
1 & 0 & \lambda_{1} \\
0 & 1 & \lambda_{2} \\
\lambda_{1} & \lambda_{2} & \lambda_{3}
\end{array}\right] .
$$

We directly compute the Christoffel symbols involved in (5.5):

$$
\Gamma_{11}^{3}=\frac{\partial_{1} \lambda_{1}}{\lambda_{3}-\left(\lambda_{1}^{2}+\lambda_{2}^{2}\right)}, \quad \Gamma_{12}^{3}=\frac{\frac{1}{2}\left(\partial_{1} \lambda_{2}+\partial_{2} \lambda_{1}\right)}{\lambda_{3}-\left(\lambda_{1}^{2}+\lambda_{2}^{2}\right)}, \quad \Gamma_{22}^{3}=\frac{\partial_{2} \lambda_{2}}{\lambda_{3}-\left(\lambda_{1}^{2}+\lambda_{2}^{2}\right)} .
$$

Hence, if $\left|\partial_{1} \lambda_{1}\right|+\left|\partial_{2} \lambda_{2}\right| \not \equiv 0$ in $\Omega$, it follows by Theorem 5.3 (iii), that the isometric immersion $y_{0}\left(x^{\prime}\right)=x^{\prime}$ of $G_{2 \times 2}=\operatorname{Id}_{2}$ is certainly not the immersion for which $\mathcal{I}_{G}\left(y_{0}\right)=0$. Of course, this does not preclude the possibility that there exists another immersion $y: \Omega \rightarrow \mathbb{R}^{3}$ of $G_{2 \times 2}$ (now necessarily non-flat), for which $\mathcal{I}_{G}(y)=0$. As we shall see below, both scenarios are possible.

(ii). Consider a subcase of (6.9), where $\lambda_{1}=0$ and $\lambda_{3}=\lambda_{2}^{2}+1$, so that:

$$
G\left(x^{\prime}, x_{3}\right)=G\left(x^{\prime}\right)=\left[\begin{array}{ccc}
1 & 0 & 0 \\
0 & 1 & \lambda_{2} \\
0 & \lambda_{2} & \lambda_{2}^{2}+1
\end{array}\right] \text {. }
$$

Consequently, by (6.10):

$$
\Gamma_{11}^{3}=0, \quad \Gamma_{12}^{3}=\frac{1}{2} \partial_{1} \lambda_{2}, \quad \Gamma_{22}^{3}=\partial_{2} \lambda_{2}
$$

We further compute:

$$
\Gamma_{11}^{1}=\Gamma_{11}^{2}=0, \quad \Gamma_{13}^{3}=\frac{1}{2} \lambda_{2} \partial_{1} \lambda_{2}, \quad \Gamma_{12}^{2}=-\frac{1}{2} \lambda_{2} \partial_{1} \lambda_{2},
$$

and:

$$
R_{112}^{3}=\partial_{1} \Gamma_{12}^{3}+\left(\Gamma_{12}^{3} \Gamma_{12}^{2}+\Gamma_{13}^{3} \Gamma_{12}^{3}\right)-\left(\Gamma_{12}^{3} \Gamma_{11}^{1}+\Gamma_{22}^{3} \Gamma_{11}^{2}\right)=\frac{1}{2} \partial_{11} \lambda_{2} .
$$

We see that when $\partial_{11} \lambda_{2} \not \equiv 0$ in $\Omega$, then $\min \mathcal{I}_{G}>0$, in view of Theorem 5.5. In particular, there is no isometric immersion of $G_{2 \times 2}$ satisfying (5.5).

(iii). Consider now a further subcase of (6.9), with $\lambda_{1}=0, \lambda_{2}=-x_{2}$ and $\lambda_{3}=x_{2}^{2}+1$, so that:

$$
G\left(x^{\prime}, x_{3}\right)=G\left(x_{2}\right)=\left[\begin{array}{ccc}
1 & 0 & 0 \\
0 & 1 & -x_{2} \\
0 & -x_{2} & x_{2}^{2}+1
\end{array}\right] .
$$

By (6.11) we get: $\Gamma_{11}^{3}=\Gamma_{12}^{3}=0$ and $\Gamma_{22}^{3}=-1$. Let $y\left(x_{1}, x_{2}\right)=\left(x_{1}, \sin x_{2}, \cos x_{2}\right)$ be an isometric immersion of $G_{2 \times 2}$ into a cylinder. This immersion has the second fundamental form $\Pi$ :

$$
\Pi=(\nabla y)^{T} \nabla \vec{N}=\left[\begin{array}{ll}
0 & 0 \\
0 & 1
\end{array}\right]=-\frac{1}{\sqrt{G^{33}}}\left[\begin{array}{ll}
\Gamma_{11}^{3} & \Gamma_{12}^{3} \\
\Gamma_{12}^{3} & \Gamma_{22}^{3}
\end{array}\right] .
$$


Hence, by Theorem 5.3 (iii) it follows that $\mathcal{I}_{G}(y)=0$.

In particular, $R_{112}^{3}=R_{221}^{3}=R_{1212} \equiv 0$ in $\Omega$. Metric $G$ is, however, nonimmersable, as a direct calculation of its scalar Ricci curvature shows:

$$
S=G^{11} R_{11}+G^{22} R_{22}+G^{33} R_{33}+2 G^{23} R_{23}=-2+2 x_{2}^{2} \not \equiv 0 .
$$

Example 6.5. In this example we will have $G_{2 \times 2}$ nonimmersable in $\mathbb{R}^{2}$. Let $\bar{\Omega} \subset\left\{\left(x_{1}, x_{2}\right) \in\right.$ $\left.\mathbb{R}^{2} ; x_{1}>x_{2}>0\right\}$ and define $G$ :

$$
G\left(x_{1}, x_{2}\right)=\left[\begin{array}{ccc}
1+x_{1}^{2} & x_{1} x_{2} & b_{1}+x_{1} b_{3} \\
x_{1} x_{2} & 1+x_{2}^{2} & b_{2}+x_{2} b_{3} \\
b_{1}+x_{1} b_{3} & b_{2}+x_{2} b_{3} & |\vec{b}|^{2}
\end{array}\right] \quad \text { where } \vec{b}=\left(-\frac{1}{3} x_{1}^{3}, \frac{1}{3} x_{2}^{3}, \frac{1}{2}\left(x_{1}^{2}-x_{2}^{2}\right)\right)^{T}
$$

We see that:

$$
y\left(x_{1}, x_{2}\right)=\left(x_{1}, x_{2}, \frac{1}{2}\left(x_{1}^{2}+x_{2}^{2}\right)\right)
$$

is an isometric immersion of $G_{2 \times 2}$ in $\mathbb{R}^{3}$. Therefore:

$$
\kappa\left(G_{2 \times 2}\right)=\frac{\partial_{11} y_{3} \partial_{22} y_{3}-\left(\partial_{12} y_{3}\right)^{2}}{\left(1+\left|\nabla y_{3}\right|^{2}\right)^{2}}=\left(1+x_{1}^{2}+x_{2}^{2}\right)^{-2} \neq 0 .
$$

By Theorem [5.3, we have: $\min \mathcal{I}_{G}=0$ iff (5.4) holds. This is equivalent to $\operatorname{sym}\left(\nabla \vec{b}_{\text {tan }}+\nabla b_{3} \otimes\right.$ $\left.\left(x_{1}, x_{2}\right)\right)=0$, and further to:

$$
\operatorname{sym}\left(\nabla b_{3} \otimes\left(x_{1}, x_{2}\right)\right)=-\operatorname{sym} \nabla \vec{b}_{t a n} .
$$

Given a scalar field $b_{3}$, there exists $\vec{b}_{\text {tan }}$ so that the above condition is satisfied iff:

$$
0=\operatorname{curl}^{T} \operatorname{curl}\left(\nabla b_{3} \otimes\left(x_{1}, x_{2}\right)\right)=-\Delta b_{3} .
$$

We see that indeed $b_{3}$ in (6.12) is harmonic, and that $\left(b_{1}, b_{2}\right)$ satisfiy: $\operatorname{sym} \nabla \vec{b}_{\text {tan }}=\operatorname{diag}\left(-x_{1}^{2}, x_{2}^{2}\right)$, which implies $\mathcal{I}_{G}(y)=0$, for the $3 \mathrm{~d}$ metric $G=Q^{T} Q$, where $Q=\left[\partial_{1} y, \partial_{2} y, \vec{b}\right]$. Note also that $\operatorname{det} Q>0$ in $\bar{\Omega}$. Hence, $G$ is given by 6.12 . One can check that $G$ is nonimmersable in $\mathbb{R}^{3}$, by

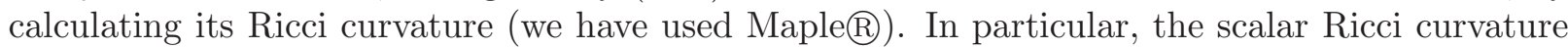
of $G$ equals:

$$
S=\sum_{i, j=1 . .3} G^{i j} R_{i j}=\frac{12}{2 x_{1}^{2}+2 x_{2}^{2}+3} \neq 0 .
$$

In section 8 we will discuss other examples of types of metric $G$, motivated by the modelling of the nematic glass.

\section{SCALing ANALYSis FOR EXAmple 6.1}

In this section we will prove the optimality of the scaling $h^{4}$ in (6.4) for every non-immersable metric tensor $G$ of the form in Example 6.1.

Theorem 7.1. Let $\lambda: \bar{\Omega} \rightarrow \mathbb{R}$ be a smooth positive function such that:

$$
M:=\nabla^{2} \lambda-\frac{1}{2 \lambda} \nabla \lambda \otimes \nabla \lambda \not \equiv 0 \quad \text { in } \Omega .
$$

Define $G\left(x^{\prime}, x_{3}\right)=G\left(x^{\prime}\right)=\operatorname{diag}\left(1,1, \lambda\left(x^{\prime}\right)\right)$. Then $G$ is non-immersable and there exist $c, C>0$ such that:

$$
c h^{4} \leq \inf E^{h} \leq C h^{4} .
$$


Proof. The equivalence of nonimmersability of $G$ with the condition $M \not \equiv 0$, as well as the upper bound has been established in Example 6.1, see (6.2) and (6.4). Hence it remains to prove the lower bound of (7.1). Recall that the minimizing isometric immersion of $G_{2 \times 2}$ here is $y\left(x^{\prime}\right)=\left(x^{\prime}, 0\right)$, whereas other quantities in the proofs of Theorems 2.1 and 3.1 are:

$$
\begin{aligned}
& \vec{b}=\sqrt{\lambda} e_{3}, \quad Q=A=\operatorname{diag}(1,1, \sqrt{\lambda}) \\
& \vec{d}=\left(-\frac{\partial_{1} \lambda}{2},-\frac{\partial_{2} \lambda}{2}, 0\right)^{T}, \quad B\left(x^{\prime}\right)=\left[\partial_{1} \vec{b}, \partial_{2} \vec{b}, \vec{d}\right]=\left[\begin{array}{ccc}
0 & 0 & -\frac{\partial_{1} \lambda}{2} \\
0 & 0 & -\frac{\partial_{2} \lambda}{2} \\
\frac{\partial_{1} \lambda}{2 \sqrt{\lambda}} & \frac{\partial_{2} \lambda}{2 \sqrt{\lambda}} & 0
\end{array}\right] .
\end{aligned}
$$

1. Let $u^{h} \in W^{1,2}\left(\Omega^{h}, \mathbb{R}^{3}\right)$ be any sequence of deformations, satisfying:

$$
E^{h}\left(u^{h}\right) \leq C h^{4} .
$$

Consider a smooth diffeomorphism $\phi: \Omega^{h_{0}} \rightarrow \mathbb{R}^{3}$ (for $h_{0}>0$ sufficiently small) given as in (6.3):

$$
\phi\left(x_{1}, x_{2}, x_{3}\right)=\left(x_{1}-\frac{x_{3}^{2}}{4} \partial_{1} \lambda, x_{2}-\frac{x_{3}^{2}}{4} \partial_{2} \lambda, \sqrt{\lambda} x_{3}\right)^{T} .
$$

We will write $\mathcal{U}^{h}=\phi\left(\Omega^{h}\right)$. Since $\nabla \phi=Q+x_{3} B-\frac{x_{3}^{2}}{4}\left(\nabla^{2} \lambda\right)^{*}$, it follows that:

$$
(\nabla \phi) A^{-1}=\operatorname{Id}_{3}+x_{3} B A^{-1}-\frac{x_{3}^{2}}{4}\left(\nabla^{2} \lambda\right)^{*}
$$

Further, noting that $B A^{-1} \in s o(3)$, by polar decomposition theorem we get:

$$
\exists R(x) \in S O(3) \quad(\nabla \phi) A^{-1}=\sqrt{(\nabla \phi) A^{-1}\left((\nabla \phi) A^{-1}\right)^{T}} R(x)=\left(\operatorname{Id}_{3}+\mathcal{O}\left(x_{3}^{2}\right)\right) R(x) .
$$

Then:

$$
\begin{aligned}
E^{h}\left(u^{h}\right) & \geq c \frac{1}{h} \int_{\Omega^{h}} \operatorname{dist}^{2}\left(\left(\nabla u^{h}\right) A^{-1}, S O(3)\right) \mathrm{d} x \\
& =c \frac{1}{h} \int_{\mathcal{U}^{h}} \operatorname{dist}^{2}\left(\nabla\left(u^{h} \circ \phi^{-1}\right)\left(\nabla h A^{-1}\right), S O(3)\right)|\operatorname{det} \nabla h|^{-1} \mathrm{~d} z \\
& \geq c \frac{1}{h} \int_{\mathcal{U}^{h}} \operatorname{dist}^{2}\left(\nabla\left(u^{h} \circ \phi^{-1}\right), S O(3)\right) \mathrm{d} z+\mathcal{O}\left(h^{4}\right),
\end{aligned}
$$

where we used that:

$\operatorname{dist}^{2}\left(F\left(\operatorname{Id}_{3}+\mathcal{O}\left(x_{3}^{2}\right)\right), S O(3)\right) \geq c \operatorname{dist}^{2}\left(F, S O(3)\left(\operatorname{Id}_{3}+\mathcal{O}\left(x_{3}^{2}\right)\right)^{-1}\right) \geq c \operatorname{dist}^{2}(F, S O(3))+\mathcal{O}\left(x_{3}^{4}\right)$.

By the geometric rigidity estimate [6], and since $\operatorname{det} \nabla \phi$ is uniformly bounded away from 0 in $\Omega$, the estimate (7.3) becomes:

$$
\begin{aligned}
E^{h}\left(u^{h}\right) & \geq c_{h} \frac{1}{h} \int_{\mathcal{U}^{h}}\left|\nabla\left(u^{h} \circ \phi^{-1}\right)-R_{h}\right|^{2} \mathrm{~d} z+\mathcal{O}\left(h^{4}\right) \geq c_{h} \frac{1}{h} \int_{\Omega^{h}}\left|\nabla u^{h}-R_{h} \nabla \phi\right|^{2}+\mathcal{O}\left(h^{4}\right) \\
& \geq c_{h} \frac{1}{h} \int_{\Omega^{h}}\left|\nabla u^{h}-R_{h}\left(Q+x_{3} B\right)\right|^{2} \mathrm{~d} x+\mathcal{O}\left(h^{4}\right),
\end{aligned}
$$

for some $R_{h} \in S O(3)$ and the constants $c_{h}$ depending on the domains $\mathcal{U}^{h}$. However, if we replace the integration on $\Omega^{h}$ in (7.3), (7.4), by integration on a small cube $\left(-\frac{h}{2}, \frac{h}{2}\right)^{3}$, all the subsequent constants, including $c_{h}$ from the geometric rigidity estimate, will be uniform and independent of $\phi$. This leads, by the well known technique of approximation [6, 14, 13], to the following result: 
Lemma 7.2. Assume (7.2). There exists a sequence of rotation fields $R^{h} \in W^{1,2}(\Omega, S O(3))$, with:

$$
\frac{1}{h} \int_{\Omega^{h}}\left|\nabla u^{h}-R^{h}\left(Q+x_{3} B\right)\right|^{2} \mathrm{~d} x \leq C h^{4} \quad \text { and } \quad \int_{\Omega}\left|\nabla R^{h}\right|^{2} \leq C h^{2} .
$$

The proof of Lemma 7.2 reproduces the lines of Theorem 1.6 in [14, hence we omit it. Note that the above is parallel to Lemma 2.3. now with an improved accuracy of the error bound due to the smaller scaling of the energy in (7.2). With the help of (7.5) we now derive the limiting properties of the sequence $u^{h}$.

2. Define $\bar{R}^{h}=\mathbb{P}_{S O(3)} f_{\Omega^{h}}\left(\nabla u^{h}\right) Q^{-1} \mathrm{~d} x$. Since: $\operatorname{dist}^{2}\left(f\left(\nabla u^{h}\right) Q^{-1}, S O(3)\right) \leq \mid f\left(\nabla u^{h}\right) Q^{-1}-$ $\left.R^{h}\left(x^{\prime}\right)\right|^{2}$ for every $x^{\prime} \in \Omega$, it follows upon integrating and using the Poincarè inequality, together with (7.5), that the projection in $\bar{R}^{h}$ is well defined. Indeed:

$$
\begin{aligned}
\operatorname{dist}^{2}\left(f_{\Omega^{h}}\left(\nabla u^{h}\right) Q^{-1}, S O(3)\right) & \leq f\left|f\left(\nabla u^{h}\right) Q^{-1} \mathrm{~d} x-R^{h}\left(x^{\prime}\right)\right|^{2} \mathrm{~d} x^{\prime} \\
& \leq C\left(f\left|f\left(\nabla u^{h}\right) Q^{-1}-f R^{h}\right|^{2}+f\left|R^{h}-f R^{h}\right|^{2}\right) \\
& \leq C\left(f_{\Omega^{h}}\left|\left(\nabla u^{h}\right) Q^{-1}-R^{h}\right|^{2}+f_{\Omega^{h}}\left|\nabla R^{h}\right|^{2}\right) \leq C h^{2} .
\end{aligned}
$$

In a similar manner as above and again by (7.5), we observe that:

$$
\begin{aligned}
& \int_{\Omega}\left|R^{h}-\bar{R}^{h}\right|^{2} \leq \int_{\Omega}\left|R^{h}-f\left(\nabla u^{h}\right) Q^{-1}\right|^{2} \leq C h^{2}, \\
& \left\|\left(\bar{R}^{h}\right)^{T} R^{h}-\operatorname{Id}_{3}\right\|_{W^{1,2}(\Omega)}^{2} \leq C h^{2} .
\end{aligned}
$$

In particular, we have the following convergence to some matrix field $S$ on $\Omega^{1}$, up to a subsequence:

$$
\frac{1}{h}\left(\left(\bar{R}^{h}\right)^{T} R^{h}-\mathrm{Id}_{3}\right) \rightarrow S \quad \text { weakly in } W^{1,2}\left(\Omega^{1}, \mathbb{R}^{3 \times 3}\right),
$$

3. Define the renormalised deformations $y^{h} \in W^{1,2}\left(\Omega^{1}, \mathbb{R}^{3}\right)$ by $y^{h}\left(x^{\prime}, x_{3}\right)=\left(\bar{R}^{h}\right)^{T} u^{h}\left(x^{\prime}, h x_{3}\right)-$ $c^{h}$, where $c^{h}$ is a constant vector such that by the Poincarè inequality and in view of:

there holds:

$$
\int_{\Omega^{1}}\left|\left(\bar{R}^{h}\right)^{T} \nabla u^{h}\left(x^{\prime}, h x_{3}\right)-Q\right|^{2} \leq C \frac{1}{h} \int_{\Omega^{h}}\left|\nabla u^{h}-\bar{R}^{h} Q\right|^{2} \leq C h^{2},
$$

$$
y^{h} \rightarrow y \quad \text { and } \quad \frac{1}{h} \partial_{3} y^{h} \rightarrow \vec{b} \quad \text { in } W^{1,2}\left(\Omega^{1}, \mathbb{R}^{3}\right) .
$$

The above is, naturally, consistent with the results of Theorem 2.1 (i). We now perform the analysis similar to step 5 in the proof of Theorem 2.1. Define the rescaled strains:

$$
\mathcal{G}^{h}\left(x^{\prime}, x_{3}\right)=\frac{1}{h^{2}}\left(\left(R^{h}\right)^{T} \nabla u^{h}\left(x^{\prime}, h x_{3}\right)-\left(Q+h x_{3} B\right)\right) A^{-1} .
$$

Note that in view of (7.5), $\left\{\mathcal{G}^{h}\right\}$ is uniformly bounded in $L^{2}$, so that up to a subsequence:

$$
\mathcal{G}^{h} \rightarrow \mathcal{G} \quad \text { weakly in } L^{2}\left(\Omega^{1}, \mathbb{R}^{3 \times 3}\right) .
$$

Consider the vector fields:

$$
\begin{aligned}
& \frac{1}{h^{2}}\left(\partial_{3} y^{h}-h\left(\vec{b}+h x_{3} \vec{d}\right)\right)=\frac{1}{h}\left(\left(\bar{R}^{h}\right)^{T} \nabla u^{h}\left(x^{\prime}, h x_{3}\right)-\left(Q+h x_{3} B\right)\right) e_{3} \\
& =\frac{1}{h}\left(\bar{R}^{h}\right)^{T}\left(\nabla u^{h}\left(x^{\prime}, h x_{3}\right)-R^{h}\left(Q+h x_{3} B\right)\right) e_{3}+\frac{1}{h}\left(\left(\bar{R}^{h}\right)^{T} R^{h}-\mathrm{Id}\right)\left(Q+h x_{3} B\right) e_{3} .
\end{aligned}
$$


The first term in the right hand side above converges in $L^{2}\left(\Omega^{1}\right)$ to 0 by (7.5), while the second term converges to $S Q e_{3}=S \vec{b}$ by (7.7). Hence we observe the same convergence of the difference quotients:

$$
\begin{aligned}
f^{s, h}\left(x^{\prime}, x_{3}\right) & =\frac{1}{h^{2}} \frac{1}{s}\left(y^{h}\left(x^{\prime}, x_{3}+s\right)-y^{h}\left(x^{\prime}, x_{3}\right)-h s\left(\vec{b}+\left(h x_{3}+\frac{h s}{2}\right) \vec{d}\right)\right) \\
& =\frac{1}{h^{2}} f_{0}^{s} \partial_{3} y^{h}\left(x^{\prime}, x_{3}+t\right)-h\left(\vec{b}+h\left(x_{3}+t\right) \vec{d}\right) \mathrm{d} t
\end{aligned}
$$

and of their $\partial_{3}$ derivatives, namely:

$$
f^{s, h} \rightarrow S \vec{b} \quad \text { and } \quad \partial_{3} f^{s, h} \rightarrow 0 \quad \text { in } L^{2}\left(\Omega^{1}, \mathbb{R}^{3}\right) .
$$

Regarding the in-plane derivatives, for $i=1,2$ we have:

$$
\begin{gathered}
\partial_{i} f^{s, h}\left(x^{\prime}, x_{3}\right) \\
=\frac{1}{h^{2} s}\left(\left(\bar{R}^{h}\right)^{T} \partial_{i} u^{h}\left(x^{\prime}, h x_{3}+h s\right)-\left(\bar{R}^{h}\right)^{T} \partial_{i} u^{h}\left(x^{\prime}, h x_{3}\right)-h s\left(\partial_{i} \vec{b}+h\left(x_{3}+\frac{s}{2}\right) \partial_{i} \vec{d}\right)\right) \\
=\frac{1}{s}\left(\left(\bar{R}^{h}\right)^{T} R^{h} \mathcal{G}^{h}\left(x^{\prime}, x_{3}+s\right)-\left(\bar{R}^{h}\right)^{T} R^{h} \mathcal{G}^{h}\left(x^{\prime}, x_{3}\right)\right) e_{i} \\
+\frac{1}{h}\left(\left(\bar{R}^{h}\right)^{T} R^{h}-\operatorname{Id}_{3}\right) B e_{i}-\left(x_{3}+\frac{s}{2}\right) \partial_{i} \vec{d} .
\end{gathered}
$$

Therefore, by (7.8) and (7.7):

$$
\partial_{i} f^{s, h} \rightarrow \frac{1}{s}\left(\mathcal{G}\left(x^{\prime}, x_{3}+s\right)-\mathcal{G}\left(x^{\prime}, x_{3}\right)\right) e_{i}+S B e_{i}-\left(x_{3}+\frac{s}{2}\right) \partial_{i} \vec{d} \quad \text { weakly in } L^{2}\left(\Omega^{1}, \mathbb{R}^{3}\right) .
$$

Concluding, $f^{s, h}$ converges weakly (up to a subsequence) in $W^{1,2}\left(\Omega^{1}\right)$ to $S \vec{b}$, and the limit in the right hand side above must coincide with $\partial_{i}(S \vec{b})$, yielding:

$$
\mathcal{G}\left(x^{\prime}, x_{3}\right) e_{i}-\mathcal{G}\left(x^{\prime}, 0\right) e_{i}=x_{3}\left(\partial_{i}(S \vec{b})-S B e_{i}\right)+\frac{x_{3}^{2}}{2} \partial_{i} \vec{d} \quad \text { for } i=1,2
$$

4. We now estimate the rescaled energies, as desired. Firstly, observe that for $\left(x^{\prime}, x_{3}\right) \in \Omega^{1}$ :

$$
W\left(\left(\nabla u^{h}\left(x^{\prime}, h x_{3}\right)\right) A^{-1}\right)=W\left(\left(R^{h}\left(x^{\prime}\right)\right)^{T}\left(\nabla u^{h}\left(x^{\prime}, h x_{3}\right)\right) A^{-1}\right)=W\left(\operatorname{Id}_{3}+h^{2} \mathcal{G}^{h}\left(x^{\prime}, x_{3}\right)+h x_{3} B\left(x^{\prime}\right) A^{-1}\right) .
$$

Since $B A^{-1} \in s o(3)$, it follows by frame invariance that:

$$
\begin{gathered}
W\left(\left(\nabla u^{h}\left(x^{\prime}, h x_{3}\right)\right) A^{-1}\right)=W\left(\left(e^{-h x_{3} B A^{-1}}\right)\left(\operatorname{Id}_{3}+h^{2} \mathcal{G}^{h}\left(x^{\prime}, x_{3}\right)+h x_{3} B\left(x^{\prime}\right) A^{-1}\right)\right) \\
=W\left(\left(\operatorname{Id}-h x_{3} B A^{-1}+\frac{h^{2} x_{3}^{2}}{2}\left(B A^{-1}\right)^{2}+\mathcal{O}\left(h^{3}\right)\right)\left(\operatorname{Id}_{3}+h^{2} \mathcal{G}^{h}\left(x^{\prime}, x_{3}\right)+h x_{3} B\left(x^{\prime}\right) A^{-1}\right)\right) \\
=W\left(\operatorname{Id}_{3}+h^{2} \mathcal{G}^{h}-\frac{h^{2} x_{3}^{2}}{2}\left(B A^{-1}\right)^{2}+\mathcal{O}(h) h^{2} \mathcal{G}^{h}+\mathcal{O}\left(h^{3}\right)\right) .
\end{gathered}
$$


Define the sets $\Omega_{h}=\left\{x \in \Omega^{1} ; h\left|\mathcal{G}^{h}(x)\right| \leq 1\right\}$. Using Taylor's expansion, we now obtain:

$$
\begin{aligned}
\liminf _{h \rightarrow 0} \frac{1}{h^{4}} E^{h}\left(u^{h}\right) & \geq \liminf _{h \rightarrow 0} \frac{1}{h^{4}} \int_{\Omega^{1}} \chi_{\Omega_{h}} W\left(\operatorname{Id}_{3}+h^{2} \mathcal{G}^{h}-\frac{h^{2} x_{3}^{2}}{2}\left(B A^{-1}\right)^{2}+\mathcal{O}\left(h^{3}\right)\right) \mathrm{d} x \\
& =\liminf _{h \rightarrow 0} \frac{1}{2} \int_{\Omega^{1}} \mathcal{Q}_{3}\left(\chi_{\Omega_{h}}\left(\mathcal{G}^{h}-\frac{x_{3}^{2}}{2}\left(B A^{-1}\right)^{2}\right) \geq \frac{1}{2} \int_{\Omega^{1}} \mathcal{Q}_{3}\left(\mathcal{G}-\frac{x_{3}^{2}}{2}\left(B A^{-1}\right)^{2}\right)\right. \\
& \geq \frac{1}{2} \int_{\Omega^{1}} \mathcal{Q}_{2}\left(\mathcal{G}\left(x^{\prime}, x_{3}\right)_{2 \times 2}+\frac{x_{3}^{2}}{2} \frac{1}{4 \lambda} \nabla \lambda \otimes \nabla \lambda\right) \mathrm{d} x,
\end{aligned}
$$

where we used the weak sequential lower-semincontinuity of the quadratic form $\mathcal{Q}_{3}$ in $L^{2}$, and since by (7.8) $\chi_{\Omega_{h}} \rightarrow 1$ in $L^{1}\left(\Omega^{1}\right)$, resulting in $\chi_{\Omega_{h}}$ sym $\mathcal{G}^{h} \rightarrow$ sym $\mathcal{G}$ weakly in $L^{2}\left(\Omega^{1}\right)$.

By (17.9) we hence arrive at:

$$
\begin{aligned}
\liminf _{h \rightarrow 0} & \frac{1}{h^{4}} E^{h}\left(u^{h}\right) \geq \frac{1}{2} \int_{\Omega^{1}} \mathcal{Q}_{2}\left(\mathcal{G}\left(x^{\prime}, 0\right)_{2 \times 2}+x_{3}(\nabla(S \vec{b})-S B)_{2 \times 2}-\frac{x_{3}^{2}}{4} M\right) \mathrm{d} x \\
\geq & \frac{1}{2} \int_{\Omega^{1}} \mathcal{Q}_{2}\left(\mathcal{G}\left(x^{\prime}, 0\right)_{2 \times 2}-\frac{x_{3}^{2}}{4} M\right) \mathrm{d} x \\
& =\frac{1}{2}\left(\int_{\Omega^{1}} \mathcal{Q}_{2}\left(\mathcal{G}\left(x^{\prime}, 0\right)_{2 \times 2}\right)-\int_{\Omega^{1}} \frac{x_{3}^{2}}{2}\left\langle L_{2}\left(\mathcal{G}\left(x^{\prime}, 0\right)_{2 \times 2}\right): M\right\rangle+\int_{\Omega^{1}} \frac{x_{3}^{4}}{16} \mathcal{Q}_{2}(M)\right) \\
& =\frac{1}{2}\left(\int_{\Omega} \mathcal{Q}_{2}\left(\mathcal{G}\left(x^{\prime}, 0\right)_{2 \times 2}\right)-\frac{1}{24} \int_{\Omega}\left\langle L_{2}\left(\mathcal{G}\left(x^{\prime}, 0\right)_{2 \times 2}\right): M\right\rangle+\frac{1}{16}\left(\frac{1}{144}+\frac{1}{180}\right) \int_{\Omega} \mathcal{Q}_{2}(M) \mathrm{d} x^{\prime}\right) \\
& =\frac{1}{2}\left(\int_{\Omega} \mathcal{Q}_{2}\left(\mathcal{G}\left(x^{\prime}, 0\right)_{2 \times 2}-\frac{1}{48} M\right) \mathrm{d} x^{\prime}+\frac{1}{16} \frac{1}{180} \int_{\Omega} \mathcal{Q}_{2}(M) \mathrm{d} x^{\prime}\right) \\
\geq & c \int_{\Omega} \mathcal{Q}_{2}(M) \mathrm{d} x^{\prime} .
\end{aligned}
$$

Above, $L_{2}: \mathbb{R}^{2 \times 2} \rightarrow \mathbb{R}^{2 \times 2}$ stands for the linear map with the property: $\mathcal{Q}_{2}(F)=\left\langle L_{2}(F): F\right\rangle$ and $\left\langle L_{2}(F): \tilde{F}\right\rangle=\left\langle L_{2}(\tilde{F}): F\right\rangle$ for all $F, \tilde{F} \in \mathbb{R}^{2 \times 2}$. The proof of Theorem 7.1 is now complete in view of the nondegeneracy of the quadratic form $\mathcal{Q}_{2}$ on symmetric matrices.

\section{Part C: Application}

\section{Applichtion to liquid crystal glass}

Nematic liquid crystals elastomers are rubber-like, cross-linked, polymeric solids, which have both positional elasticity, due to rubber-like, solid response of the polymer chains, and the orientation elasticity due to the separately deforming director. A nematic liquid crystal glass is a very highly cross-linked nematic liquid crystal elastomer such that the director is effectively constrained to move with the liquid crystal elastomer matrix.

In this section we consider a model of nematic glass [23, 19] whose referential conformation $A$ corresponds to a prolate ellipsoid, elongating the eigenvector $\vec{n}$ by the factor $\lambda$, while shrinking the invariant $2 \mathrm{~d}$ subspace $\vec{n}^{\perp}=\operatorname{span}(v, w)$ by factor $\lambda^{\nu}$ :

$$
A=\lambda^{-\nu} v \otimes v+\lambda^{-\nu} w \otimes w+\lambda \vec{n} \otimes \vec{n}=\lambda^{-\nu}\left(\operatorname{Id}_{3}+\left(\lambda^{\nu+1}-1\right) \vec{n} \otimes \vec{n}\right), \quad \lambda>1, \quad|\vec{n}|=1 .
$$

In other circumstances, $A$ corresponds to a contraction $\lambda$ in direction of $\vec{n}$ and an expansion $\lambda^{-\nu}$ in the perpendicular directions, and so $\lambda$ could also be less than 1 [20]. The coefficient $\nu$ is 
experimentally verified to be in the range $\frac{1}{2}<\nu<2$. Setting $r=\lambda^{\nu+1}$, and writing $\lambda^{-\nu}=r^{\delta}$ with $\delta=-\frac{\nu}{\nu+1}$, we obtain the metric $G$ and its symmetric square root $A=\sqrt{G}$ given by:

$$
G\left(x^{\prime}, x_{3}\right)=G\left(x^{\prime}\right)=r^{2 \delta}\left(\operatorname{Id}_{3}+\left(r^{2}-1\right) \vec{n} \otimes \vec{n}\right), \quad A\left(x^{\prime}\right)=r^{\delta}\left(\operatorname{Id}_{3}+(r-1) \vec{n} \otimes \vec{n}\right) .
$$

We start by the following observation:

Theorem 8.1. Assume that:

$$
\vec{n} \in S^{1} \quad \text { i.e. } \vec{n}=\left(n_{1}, n_{2}, 0\right)^{T} \in S^{2} \text {, with } n=\left(n_{1}, n_{2}\right) \in S^{1} .
$$

Then the following conditions are equivalent:

(i) the metric $G$ as in (8.1) is immersable, i.e. $G=(\nabla u)^{T} \nabla u$ for some smooth $u: \Omega^{1} \rightarrow \mathbb{R}^{3}$,

(ii) the Gaussian curvature $\kappa\left(\operatorname{Id}_{2}+\left(r^{2}-1\right) n \otimes n\right)$ vanishes identically in $\Omega$,

(iii) $\operatorname{curl}^{T} \operatorname{curl} G_{2 \times 2}=0$,

(iv) the following curvatures of $G$ vanish: $R_{112}^{3}=R_{221}^{3}=R_{1212}=0$.

Proof. It is clear that (i) holds iff the Riemann tensor of $G$ vanishes, which is equivalent to:

$$
\kappa=\kappa\left(\operatorname{Id}_{2}+\left(r^{2}-1\right) n \otimes n\right)=0 .
$$

We now calculate the Gaussian curvature $\kappa$ of the $2 \mathrm{~d}$ metric $\operatorname{Id}_{2}+\left(r^{2}-1\right) n \otimes n$ and prove that:

$$
\kappa=r^{2 \delta} \kappa\left(G_{2 \times 2}\right)=\frac{1}{2}\left(r^{-2}-1\right) \operatorname{curl}^{T} \operatorname{curl}(n \otimes n) .
$$

This will achieve the lemma, because $R_{112}^{3}=R_{221}^{3}=0$ automatically, while $R_{1212}=0$ is equivalent to (ii). We write $r^{2}-1=\gamma>0$ and compute:

$$
\begin{aligned}
\left(\operatorname{det}\left(\operatorname{Id}_{2}+\gamma n \otimes n\right)\right)^{2} \kappa= & \operatorname{det}\left[\begin{array}{cccc}
q & \frac{1}{2} e_{, 1} & f_{, 1}-\frac{1}{2} e_{, 2} \\
f_{, 2}-\frac{1}{2} g_{, 1} & e & f \\
\frac{1}{2} g_{, 2} & f & g
\end{array}\right] \\
& -\operatorname{det}\left[\begin{array}{ccc}
0 & \frac{1}{2} e_{, 2} & g, 1 \\
\frac{1}{2} e_{, 2}-\frac{1}{2} g_{, 1} & e & f \\
\frac{1}{2} g_{, 1} & f & g
\end{array}\right],
\end{aligned}
$$

where $q=-\frac{1}{2} e_{, 22}+f_{, 12}-\frac{1}{2} g, 11$ and $e=1+\gamma n_{1}^{2}, f=\gamma n_{1} n_{2}, g=1+\gamma n_{2}^{2}$. A direct calculation now gives that the right hand side above equals:

$$
\begin{aligned}
(1+\gamma) q+\gamma^{3} \cdot 0+\gamma^{2}( & -n_{2}^{2} n_{1,1} n_{2,2}-n_{1} n_{2} n_{2,1} n_{2,2}+n_{1} n_{2} n_{1,2} n_{2,2}-n_{1} n_{2} n_{1,1} n_{1,2} \\
& \left.-n_{1}^{2} n_{1,1} n_{2,2}+n_{1} n_{2} n_{1,1} n_{2,1}+n_{2}^{2} n_{2,1}^{2}+n_{1}^{2} n_{1,2}^{2}\right)=(1+\gamma) q .
\end{aligned}
$$

The equality above follows since all the terms in the bracket multiplying $\gamma^{2}$ cancel out. This can be easily seen by substituting $\left(n_{1}, n_{2}\right)=(\cos \theta, \sin \theta)$ for some angle function $\theta: \Omega \rightarrow \mathbb{R}$.

We hence see that $\kappa=0$ iff $q=0$. But note that:

$$
q=-\frac{\gamma}{2}\left(\left(n_{1}^{2}\right)_{, 22}-2\left(n_{1} n_{2}\right)_{, 12}+\left(n_{2}^{2}\right)_{, 11}\right)=-\frac{\gamma}{2} \operatorname{curl}^{T} \operatorname{curl}(n \otimes n) .
$$

Since $\operatorname{det}\left(\operatorname{Id}_{2}+\left(r^{2}-1\right) n \otimes n\right)=1+\gamma$, it follows that $\kappa=-\frac{1}{2} \frac{\gamma}{\gamma+1} \operatorname{curl}^{T} \operatorname{curl}(n \otimes n)$. 
Example 8.2. In accordance with [8.1), the following metric has been put forward in [18] for the description of disclination-mediated thermo-optical response in nematic glass sheets: $G\left(x^{\prime}, x_{3}\right)=$ $\alpha \operatorname{Id}_{3}+\beta \vec{n}\left(x^{\prime}\right) \otimes \vec{n}\left(x^{\prime}\right)$, where $\alpha, \beta>0$ are constants, and $\vec{n}$ is as in (8.2) with:

$$
n_{1}=\cos (\theta+\psi), \quad n_{2}=\sin (\theta+\psi), \quad \theta=\arctan \frac{x_{2}}{x_{1}}, \quad \psi \equiv \text { const } .
$$

Note that $\theta$ is the polar angle and so setting the constant $\psi=0$ gives the radial pattern, while $\psi=\pi / 2$ gives the azimuthal pattern, and other values of $\psi$ yield spiral patterns. It is easy to check that $\operatorname{curl}^{T} \operatorname{curl}(n \otimes n)=0$. Therefore, if the simply connected $\Omega$ does not contain 0 (since 0 is a singularity for $G$ ), then the metrics $G_{2 \times 2}$ and $G$ are immersable by Lemma 8.1, and thus $\inf E^{h}=0$. However, one may not have a global immersion (implying hence a higher energy scaling - see [19] for a construction with $h^{2}$ scaling) if $0 \in \Omega$.

Remark 8.3. Consider any 2 d metric $G_{2 \times 2}$ with constant eigenvalues $0<\lambda_{1} \leq \lambda_{2}$ :

$$
G_{2 \times 2}=\lambda_{1} v \otimes v+\lambda_{2} w \otimes w=\lambda_{1}\left(\operatorname{Id}_{2}-\frac{\lambda_{2}-\lambda_{1}}{\lambda_{1}} w \otimes w\right) .
$$

We see that such $G_{2 \times 2}$ is flat iff $\operatorname{curl}^{T} \operatorname{curl}\left(G_{2 \times 2}\right)=0$. Interestingly, $\operatorname{curl}^{T}$ curl is the leading order term in the expansion of the Gaussian curvature of a $2 \mathrm{~d}$ metric at $\operatorname{Id}_{2}$.

In the $2 \mathrm{~d}$ case as in (8.2), we directly obtain:

Theorem 8.4. Assume that $G$ is as in (8.1) with 8.2). Then, Theorems 2.1 and 3.1 hold with the Cosserat vector $\vec{b}$ given by:

$$
\vec{b}=r^{\delta} \vec{N}
$$

and with the limiting functional:

$$
\begin{aligned}
\mathcal{I}_{G}(y)=\mathcal{I}_{\vec{n}}(y) & =\frac{1}{24} \int_{\Omega} \mathcal{Q}_{2}\left(x^{\prime}, r^{\delta}(\nabla y)^{T} \nabla \vec{N}\right) \mathrm{d} x^{\prime} \\
& =\frac{1}{24} \int_{\Omega} \mathcal{Q}_{2}^{0}\left(r^{\delta}\left(A_{2 \times 2}\right)^{-1}(\nabla y)^{T} \nabla \vec{N}\left(A_{2 \times 2}\right)^{-1}\right) \mathrm{d} x^{\prime} .
\end{aligned}
$$

Denote:

$$
\alpha=\frac{r-1}{r} .
$$

Then:

$$
\left(A_{2 \times 2}\right)^{-1}=\frac{1}{r^{1+\delta}}\left(\operatorname{Id}_{2}+(r-1) n^{\perp} \otimes n^{\perp}\right)=\frac{1}{r^{\delta}}\left(\operatorname{Id}_{2}-\alpha n \otimes n\right)
$$

and the quadratic form in the second integrand in (8.4) can be equivalently expressed as:

$$
\begin{aligned}
r^{\delta}\left(A_{2 \times 2}\right)^{-1} & (\nabla y)^{T} \nabla \vec{N}\left(A_{2 \times 2}\right)^{-1} \\
& =r^{-\delta}\left((\nabla y)^{T} \nabla \vec{N}-2 \alpha \operatorname{sym}\left(\left(n \otimes \partial_{n} y\right) \nabla \vec{N}\right)+\alpha^{2}\left\langle\partial_{n} y, \partial_{n} \vec{N}\right\rangle n \otimes n\right) .
\end{aligned}
$$

Moreover, when $W$ is isotropic so that (4.4) holds, we have:

$$
\forall F_{2 \times 2} \in \mathbb{R}_{\text {sym }}^{2 \times 2} \quad \mathcal{Q}_{2}\left(x^{\prime}, F_{2 \times 2}\right)=\frac{1}{r^{4 \delta}} \mathcal{Q}_{2, i s o}^{0}\left(\left(\operatorname{Id}_{2}-\alpha n \otimes n\right) F_{2 \times 2}\left(\operatorname{Id}_{2}-\alpha n \otimes n\right)\right)
$$

We now turn to the general case of the general 3d director $\vec{n}$. 
Theorem 8.5. Assume that $G$ is of the form (8.1). Let $n=\left(n_{1}, n_{2}\right) \in \mathbb{R}^{2}$ denote the tangential component of the director vector $\vec{n}$. Denote also:

$$
\gamma=\frac{1}{n_{3}^{2}+|n|^{2} r^{2}}
$$

Then Theorems [2.1 and 3.1 hold with the Cosserat vector $\vec{b}$ is given by:

$$
\vec{b}=\left(r^{2}-1\right) n_{3} \gamma\left(\partial_{n} y\right)+r \sqrt{\gamma}\left(r^{\delta} \vec{N}\right)=\frac{\left(r^{2}-1\right) n_{3}}{n_{3}^{2}+|n|^{2} r^{2}} \partial_{n} y+\frac{r^{1+\delta}}{\sqrt{n_{3}^{2}+|n|^{2} r^{2}}} \vec{N}
$$

and with the limiting functional:

$$
\mathcal{I}_{G}(y)=\mathcal{I}_{\vec{n}}(y)=\frac{1}{24} \int_{\Omega} \mathcal{Q}_{2}\left(x^{\prime},(\nabla y)^{T} \nabla \vec{b}\right) \mathrm{d} x^{\prime} .
$$

Moreover, when $W$ is isotropic so that (4.4) holds, we have:

$$
\begin{aligned}
\forall F_{2 \times 2} \in \mathbb{R}_{\text {sym }}^{2 \times 2} \quad & \mathcal{Q}_{2}\left(x^{\prime}, F_{2 \times 2}\right)= \\
& =\mu \frac{1}{r^{4 \delta}}\left(\left|F_{2 \times 2}\right|^{2}-2\left(\left(r^{2}-1\right) \gamma\right)\left|F_{2 \times 2} n\right|^{2}+\left(\left(r^{2}-1\right) \gamma\right)^{2}\left\langle F_{2 \times 2} n, n\right\rangle^{2}\right) \\
& +\frac{\lambda \mu}{\lambda+\mu} \frac{1}{r^{4 \delta}}\left(\operatorname{tr} F_{2 \times 2}-\left(\left(r^{2}-1\right) \gamma\right)\left\langle F_{2 \times 2} n, n\right\rangle\right)^{2} .
\end{aligned}
$$

Setting $\tilde{\gamma}=\frac{1}{|n|^{2}}(1-\sqrt{\gamma})$, the above formula is equivalent to:

$$
\mathcal{Q}_{2}\left(x^{\prime}, F_{2 \times 2}\right)=\frac{1}{r^{4 \delta}} \begin{cases}\mathcal{Q}_{2, i s o}^{0}\left(\left(\operatorname{Id}_{2}-\tilde{\gamma} n \otimes n\right) F_{2 \times 2}\left(\operatorname{Id}_{2}-\tilde{\gamma} n \otimes n\right)\right) & \text { if } n_{3}\left(x^{\prime}\right)^{2}<1, \\ \mathcal{Q}_{2, i s o}^{0}\left(F_{2 \times 2}\right) & \text { if } n_{3}\left(x^{\prime}\right)^{2}=1 .\end{cases}
$$

Proof. We easily compute:

$$
\begin{aligned}
& \operatorname{det} G=r^{2+6 \delta}, \quad \operatorname{det} G_{2 \times 2}=r^{4 \delta}\left(r^{2}-\left(r^{2}-1\right) n_{3}^{2}\right) \\
& \left(G_{2 \times 2}\right)^{-1}=\frac{1}{r^{2 \delta}}\left(n_{3}^{2}+|n|^{2} r^{2}\right)^{-1}\left(\operatorname{Id}-\left(r^{2}-1\right) n^{\perp} \otimes n^{\perp}\right),
\end{aligned}
$$

where $n^{\perp}=\left(n_{1}, n_{2}\right)^{\perp}=\left(-n_{2}, n_{1}\right)$. Therefore:

$$
\left(G_{2 \times 2}\right)^{-1}\left[\begin{array}{l}
G_{13} \\
G_{23}
\end{array}\right]=\frac{\left(r^{2}-1\right) n_{3}}{n_{3}^{2}+|n|^{2} r^{2}} n
$$

which implies the formula (8.4).

To prove (8.6) in view of Theorem 4.3, it is now enough to check directly that the positive definite matrix $r^{-\delta}\left(\operatorname{Id}_{2}-\tilde{\gamma} n \otimes n\right)$ equals ${\sqrt{G_{2 \times 2}}}^{-1}$. Indeed:

$$
\begin{aligned}
\left(\mathrm{Id}_{2}-\tilde{\gamma} n \otimes n\right)^{2}\left(\mathrm{Id}_{2}+\left(r^{2}-1\right) n \otimes n\right) & =\left(\mathrm{Id}_{2}+\left(\tilde{\gamma}|n|^{2}-2 \tilde{\gamma}\right) n \otimes n\right)\left(\operatorname{Id}_{2}+\left(r^{2}-1\right) n \otimes n\right) \\
& =\left(\mathrm{Id}_{2}-\left(r^{2}-1\right) \gamma n \otimes n\right)\left(\operatorname{Id}_{2}+\left(r^{2}-1\right) n \otimes n\right)=\mathrm{Id},
\end{aligned}
$$

as $\tilde{\gamma}^{2}|n|^{2}-2 \tilde{\gamma}=-\left(r^{2}-1\right) \gamma$.

Remark 8.6. The expression in (8.3) is consistent with (8.6), as for $n_{3}=0$ it follows that $\gamma=\frac{r^{2}-1}{r^{2}}$ and $\tilde{\gamma}=1-1 / r=\alpha$. The expression in (8.3) is also consistent with Remark 4.4, in 
the following sence. Take $\vec{n}=e_{3}$. Then $D=r^{-2 \delta} \operatorname{diag}\left(1,1, r^{-1}\right) F_{2 \times 2}^{*} \operatorname{diag}\left(1,1, r^{-1}\right)=r^{-2 \delta} F_{2 \times 2}^{*}$. Hence, by (4.10):

$$
\mathcal{Q}_{2}\left(x^{\prime}, F_{2 \times 2}\right)=\frac{1}{r^{4 \delta}}\left(\mu\left|F_{2 \times 2}\right|^{2}+\frac{\lambda \mu}{\lambda+\mu}\left|\operatorname{tr} F_{2 \times 2}\right|^{2}\right),
$$

while (8.5), (8.6) give the same formula directly.

\section{REFERENCES}

[1] B. Barker, M. Lewicka and K. Zumbrun, Existence and stability of viscoelastic shock profiles, Arch. Rational Mech. Anal. 200, Number 2, (2011) 491-532.

[2] J.F. Borisov, $\mathcal{C}^{1, \alpha}$-isometric immersions of Riemannian spaces, Doklady 163, (1965) 869-871.

[3] Y-C. Chen and E. Fried, Uniaxial nematic elastomers: constitutive framework and a simple application, Proc. R. Soc. A 462, (2006) 1295-1314.

[4] S. Conti, C. De Lellis and L. Szkelyhidi Jr., h-principle and rigidity for $\mathcal{C}^{1, \alpha}$ isometric embeddings, Nonlinear Partial Differential Equations Abel Symposia, Volume 7 (2012), 83-116.

[5] G. Friesecke, R. James, M.G. Mora and S. Müller, Derivation of nonlinear bending theory for shells from three-dimensional nonlinear elasticity by Gamma-convergence, C. R. Math. Acad. Sci. Paris, 336 (2003), no. 8, 697-702.

[6] G. Friesecke, R. James and S. Müller, A hierarchy of plate models derived from nonlinear elasticity by gamma-convergence, Arch. Ration. Mech. Anal., 180 (2006), no. 2, 183-236.

[7] M. Gromov, Partial Differential Relations, Springer-Verlag, Berlin-Heidelberg, (1986).

[8] M. Gurtin, E. Fried and L. Anand, The mechanics and thermodynamics of continua, Cambridge University Press, Cambridge, 2010.

[9] Q. Han and J.X. Hong, Isometric embedding of Riemannian manifolds in Euclidean spaces, Mathematical Surveys and Monographs, 130 American Mathematical Society, Providence, RI (2006).

[10] P. Hornung, M. Lewicka and R. Pakzad, Infinitesimal isometries on developable surfaces and asymptotic theories for thin developable shells, Journal of Elasticity, 111, Number 1 (2013)

[11] Y. Klein, E. Efrati and E. Sharon, Shaping of elastic sheets by prescription of non-Euclidean metrics, Science, 315 (2007), 1116-1120.

[12] H. Le Dret., and A. Raoult, The Nonlinear Membrane Model as Variational Limit of Nonlinear ThreeDimensional Elasticity, Journal De Mathmatiques Pures Et Appliques 74 (1995): 549 ?578.

[13] M. Lewicka and R. Pakzad, Scaling laws for non-Euclidean plates and the $W^{2,2}$ isometric immersions of Riemannian metrics, ESAIM: Control, Optimisation and Calculus of Variations, 17, no 4 (2011), 1158-1173.

[14] M. Lewicka, L. Mahadevan and R. Pakzad, The Foppl-von Karman equations for plates with incompatible strains, Proceedings of the Royal Society A 467 (2011), 402-426.

[15] M. Lewicka, L. Mahadevan and R. Pakzad, The Monge-Ampere constrained elastic theories of shallow shells, submitted.

[16] M. Lewicka, M.G. Mora and R. Pakzad, The matching property of infinitesimal isometries on elliptic surfaces and elasticity of thin shells, Arch. Rational Mech. Anal. (3), 200 (2011), 1023-1050.

[17] F. C. Liu, A Lusin property of Sobelov functions, Indiana U. Math. J. 26 (1977) 645-651.

[18] C.D. Modes, K. Bhattacharya and M. Warner, Disclination-mediated thermo-optical response in nematic glass sheets, Phys. Rev. E $\mathbf{8 1}$ (2010).

[19] C.D. Modes, K. Bhattacharya and M. Warner, Gaussian curvature from flat elastica sheets, Proc. Roy. Soc. A 467 (2011) 1121-1140.

[20] C.D. Modes and M. Warner, Blueprinting nematic glass: Systematically constructing and combining active points of curvature for emergent morphology, 84 (2011), 021711-1-7.

[21] N. Kuiper, On $\mathcal{C}^{1}$ isometric imbeddings i,ii, Proc. Kon. Acad. Wet. Amsterdam A 58, (1955) 545-556, $683-689$

[22] J.C. Nash, $\mathcal{C}^{1}$ isometric imbeddings, Ann. Math. 60, (1954) 383-396.

[23] M. Warner and E. Terentjev, Liquid crystal elastomers, Oxford University Press (2003).

Kaushik Bhattacharya, Division of Engineering and Applied Science, California Institute of TechNOLOGy, PASADENA, CA 91125 
Marta Lewicka, University of Pittsburgh, Department of Mathematics, 139 University Place, Pittsburgh, PA 15260

Mathias Schäffner, University of Würzburg, Institute for Mathematics, Emil-Fischer-Str. 40, 97074 Würzburg, Germany

E-mail address: bhatta@caltech.edu, lewicka@pitt.edu, mathias.schaeffner@mathematik. uni-wuerzburg.de 